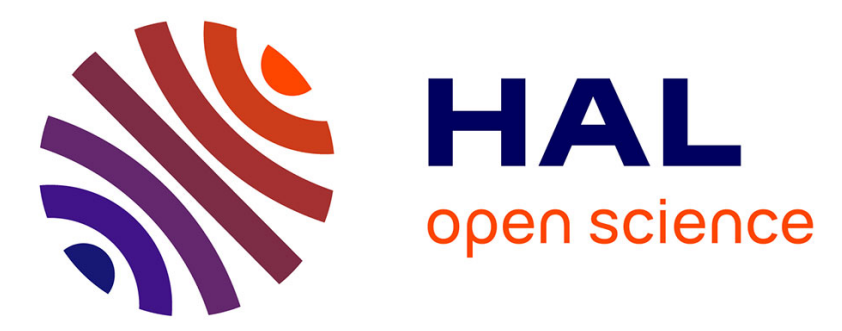

\title{
Measurements and modeling of the gas flow in a microchannel: influence of aspect ratios, surface nature, and roughnesses
}

Pierre Perrier, Mustafa Hadj-Nacer, J. Gilbert Meolans, Irina Martin Graur

\section{- To cite this version:}

Pierre Perrier, Mustafa Hadj-Nacer, J. Gilbert Meolans, Irina Martin Graur. Measurements and modeling of the gas flow in a microchannel: influence of aspect ratios, surface nature, and roughnesses. Microfluidics and Nanofluidics, 2019, 23 (97), 10.1007/s10404-019-2259-1 . hal-02904989

\section{HAL Id: hal-02904989 \\ https://hal-amu.archives-ouvertes.fr/hal-02904989}

Submitted on 22 Jul 2020

HAL is a multi-disciplinary open access archive for the deposit and dissemination of scientific research documents, whether they are published or not. The documents may come from teaching and research institutions in France or abroad, or from public or private research centers.
L'archive ouverte pluridisciplinaire HAL, est destinée au dépôt et à la diffusion de documents scientifiques de niveau recherche, publiés ou non, émanant des établissements d'enseignement et de recherche français ou étrangers, des laboratoires publics ou privés. 


\section{Measurements and modeling of the gas flow in a 2 microchannel: influence of aspect ratios, surface nature

\section{Abstract}

This article extends in various directions our previous studies related to gas flow in long rectangular cross-section microchannels. In the present article, the mass flow rate of various gases through microchannels with different aspect ratios and, various surface coatings $\left(\mathrm{Au}\right.$ and $\mathrm{SiO}_{2}$ ) and surface roughnesses (from 0.9 to $12 \mathrm{~nm}$ ) is measured under isothermal conditions. Previously, we developed a method to calculate the mass flow rate through rectangular microchannels that allows taking into account the real dimensions of the rectangular channel cross-section. In the present article, this method was applied to extract the velocity slip and tangential momentum accommodation coefficients in the frame of the Maxwell diffuse-specular scattering kernel. An extension of the previous approach is also proposed in the present paper. This extension allows considering the possible difference in properties (roughness or material) between the vertical and horizontal channel walls by introducing different accommodation coefficients for each wall. By applying the new method, we can extract a single accommodation coefficient for all the channel walls under the assumption of homogeneous material and roughness and two different accommodation coefficients for the horizontal and vertical walls in the case when the two walls have different properties (roughness or material).

\section{Introduction}

With arriving of MEMS technologies, a great diversity appears in the manufacturing of micro-systems. These new possibilities widen the choice of materials for a micro-systems substrate as well as of deposit layers and thus allow the extension of the micro-systems functionalities. All these micro-devices have the same characteristic property: their surface-to-volume ratio is much larger compared to the conventional devices. Therefore, the gas-surface interaction affects the flow characteristics considerably in such micro-systems.

For several decades, the influence of the surface nature and surface roughness on the flow properties at small scale was studied experimentally, see for example 
the review papers [1], [2]. However, only a few experimental works explored the gas/wall interaction by varying the nature and the roughness of the walls [3], [4], [5], [6]. The authors of Refs. [3], [6] studied the roughness influence and reported smaller values of the Tangential Momentum Accommodation Coefficient (TMAC) for rough surfaces compared to that of smooth surfaces for both monoatomic and polyatomic gases. However, the opposite trend was reported in Ref. [4], where a large value of TMAC and substantial backscattering with surfaces of large roughness (cooper, $5 \mu \mathrm{m}$ grooves) were measured, as compared to sapphire, glass, and electronically polished gold surfaces. The authors of Ref. [5] also reported larger values of TMAC for rough surfaces compared to polished steel spheres surfaces. From an experimental point of view, the difficulty of this kind of studies lies notably in the correct consideration of the fabrication process and in the precise geometrical characterization of studied systems.

Recently, several numerical studies have been performed, where the surface roughness was simulated by regular structures of various shapes. These simulations were carried out in the frame of different approaches: the continuum modeling (Navier-Stokes equations), [7], [8], the DSMC modeling [9] and the kinetic modeling (S-model) [10]. The influence of the random roughness was studied by using the Molecular Dynamics (MD) simulations in Refs. [11], [12]. The random roughness was also simulated by Weierstrass-Mandelbrot fractal function by applying a linearized BGK equation and the generalized Reynolds equation [13]. A significant influence of the surface roughness was found in the case of the Poiseuille and Couette flows [13]. A different approach was proposed by the authors of Ref. [14]: new scattering kernel allowed to incorporate the roughness effect directly in the boundary conditions. Despite numerous studies, the problem is far to be completely resolved, and new experimental data are needed to characterize gas-surface interaction.

Extending the topics of previous studies, [15], [16], [17], experimental and theoretical analysis of the influence of the surface roughness and the surface nature on gas flow properties at small scale is realized in this work. This analysis allows us to enhance the understanding of the gas-solid surface interaction by considering the behaviors of the same gases on different surfaces. The rectangular microchannels used in this work are fabricated by silicon etching technology. The internal surfaces of these channels are covered by a gold layer of different roughnesses or by a layer of silica. The relatively moderate roughness $(\approx 0.1 \%$ of the channel high) was chosen to avoid affecting the rectangular shape of channel cross-section. In this work, we analyze and quantify the influence of various factors on the flow properties: the gas itself, the wall materials, the solid surface roughness and the height/wide ratio, characterizing the geometry of the channel cross-sections.

Finally, we present a brief summary of a complete analytical modeling of flow in the slip regime that takes into account the two lateral channel dimensions. By comparing experimental values and analytical results, we deduce various values of the tangential momentum accommodation coefficient, by assuming that it is not the same for the horizontal and vertical walls. Indeed, in the fabrication process, the roughness of the vertical channel walls is not measured. Therefore, 
we extend our bi-dimensional treatment by introducing different accommodation coefficients for the vertical and horizontal walls.

\section{Microchannel Properties and Experimental Setup}

In this section, short description of the method used to fabricate the microchannels is provided. Then, the experimental technique used for the measurements of the mass flow rate through the microchannels is briefly introduced.

\subsection{Microchannels Fabrication Summary}

The details of the microchannel fabrication process are shown in Figure 1. First, a silicon wafer is prepared and cleaned to remove any metal or organic contaminations from the surface. Then, a drop of photoresist resin solution (SPR 220) is deposed in the center of the silicon wafer. The wafer is then spun at high speed between 500 to $5000 \mathrm{rpm}$ for 30 to 60 seconds. The rotation speed and the viscosity of the resin solution determine the resulting thickness of the photoresist resin layer. After spinning, the silicon wafer is heated to evaporate the solvent from the resin solution to form a solid resin layer ( $\operatorname{step} a$ ). Second, the photoresist resin is exposed to UV light through a mask to define the shape of the channels. This procedure allows to obtain two parts: exposed and unexposed regions. The exposed resin is then removed (step $b$ ). The next step (c) is the Deep Reactive-Ion Etching (DRIE) [18], [19], [20] in the parts where the resin is removed. During etching the silicon wafer is attacked with Argon ions $\left(A r^{+}\right)$jet that strikes the surface perpendicularly, removing the material until achieving the desired half depth $(h / 2)$ of the microchannel. After etching step a layer of gold $(\mathrm{Au})$ or silica $\left(\mathrm{SiO}_{2}\right)$ is generated on the wafer surfaces, by cathodic sputtering for gold deposition and by oxidation for silicon dioxide deposition (step $d$ ). Then, the resin remaining from the first development is stripped off and the layer of gold or silica is attacked with ions to obtain the desired roughness of the surface ( $\operatorname{step} e$ ). Another layer of photoresist resin is deposed again on the wafer by sputtering technique (step $f$ ). The photoresist resin is exposed to the UV light and the resin layer is removed in the nonetched regions (step $\mathrm{g}$ ). Finally, a layer of gold of $100 \mathrm{~nm}$ is deposed on the wafer to be used in the bonding step ( resin is stripped away (step $i$ ). The last step is the wafer bonding: the wafers fabricated with the same width $(w)$ and depth $(h / 2)$ are assembled together using the thermocompression technique at the eutectic point $(636 \mathrm{~K}, 2.85 \% \mathrm{Si}$ and $97.15 \% A u$ ) by applying a moderate pressure on the wafers at a temperature of $573 K$ (step $j)$. After the fabrication steps the assembled wafers are cut by a precise saw in order to obtain the microchannels.

The rectangular microchannels, used in the present study, were fabricated in two different ways. First, for the channels of A group and S1 channel, two identical silicon wafers with a cavity of $h / 2$ depth, etched using the DRIE technique, are bounded together, Fig. 1((j) Wafers bonding channels A and $S 1)$. Second, for the E and $S 2-S 4$ channels, a wafer with a cavity of $h$ depth, etched using 


\begin{tabular}{cccccc}
\hline \hline Channel & Material & Roughness $\left[10^{-9} \mathrm{~m}\right]$ & $h\left[10^{-6} \mathrm{~m}\right]$ & $w\left[10^{-6} \mathrm{~m}\right]$ & $L\left[10^{-3} \mathrm{~m}\right]$ \\
\hline \hline$A 1$ & $A u$ & 0.9 & 27.8 & $52.2 \pm 0.5$ & 15.07 \\
$A 2$ & & 0.9 & 27.6 & $107.6 \pm 0.5$ & 15.00 \\
$A 3$ & & 0.9 & 27.9 & $504.0 \pm 1.5$ & 15.06 \\
$A 4$ & & 1.1 & 25.8 & $1005.5 \pm 3.0$ & 14.87 \\
\hline 1 & $A u$ & 12.0 & 33.5 & $55.5 \pm 0.5$ & 15.02 \\
$E 2$ & & 12.0 & 35.2 & $103.8 \pm 0.5$ & 15.19 \\
$E 3$ & & 12.0 & 34.9 & $505.0 \pm 1.5$ & 15.22 \\
$E 4$ & & 12.0 & 34.2 & $1001.3 \pm 3.0$ & 15.06 \\
\hline$S 1$ & $S_{i} O_{2}$ & 1.1 & 24.3 & $50.1 \pm 0.5$ & 13.68 \\
$S 2$ & & 1.1 & 42.3 & $100.0 \pm 0.5$ & 15.06 \\
$S 3$ & & 1.1 & 42.0 & $500.0 \pm 1.5$ & 15.06 \\
$S 4$ & & 1.1 & 41.5 & $1000.0 \pm 3.0$ & 15.06 \\
\hline \hline
\end{tabular}

Table 1: Dimensions of the rectangular microchannels. The uncertainty of the microchannel height $h$ measurements is $\pm 0.5 \mu \mathrm{m}$, the uncertainty on the measurements of the microchannel width, $w$, depends on the channel width value and it is provided in Table; the uncertainty on the measurements of the channel length $L$ is equal to $\pm 0.1 \mathrm{~mm}$.

the DRIE technique, is bounded with a flat wafer, Fig. 1((j) Wafers bonding channels $S 2-S 4 \& \mathrm{E}$ ).

\subsection{Microsystems Description}

All the microchannels, fabricated using the technique, described in the previous Section, have rectangular cross-sections of different aspect ratios. They were divided into three groups noted by letters $\mathrm{A}, \mathrm{E}$ and $\mathrm{S}$ according to the surface coating and surface roughness:

- The first group, referenced with letter A, corresponds to the microchannels coated using a gold layer $(\mathrm{Au})$ on all internal surfaces with a mean roughness of $1 \mathrm{~nm}^{1}$.

- The second group, referenced with letter E, corresponds to the microchannels coated also with a layer of gold on all internal surfaces, but with a mean roughness of $12 \mathrm{~nm}$.

- The third group, referenced with the letter S, corresponds to the microchannels coated with silica $\left(\mathrm{SiO}_{2}\right)$ layer on all internal surfaces with a mean roughness of $1.1 \mathrm{~nm}$.

The microchannels that are in the same group differ only by their aspect ratio. Table 1 summarizes the properties and dimensions of the microchannels.

\footnotetext{
${ }^{1}$ Root Mean Square (RMS)
} 
The uncertainty on the microchannel height and width measurements are related essentially to the uncertainty on the micro-ruler used to measure the cross-section dimensions and to some eventual other errors related to the quality and sharpness of the pictures taken with the microscope. The uncertainty on the microchannel height is estimated as $\pm 0.5 \mu \mathrm{m}$, see Table 1 . The uncertainty on the microchannel width depends on the average width of the microchannel and it is provided in Table 1 . This uncertainty variation is related to the zoom used to take the pictures of the microchannels cross-section. The uncertainty on the microchannels length is only related to the caliper uncertainty and it is equal to $\pm 0.1 \mathrm{~mm}$.

The roughness of the microchannels was measured using a profilometer (Alpha-Step IQ) before the bonding step of the two wafers. The measurements are made in three sections: beginning, center and end of the microchannel. Figure 2 shows an example of roughness measurement made for the microchannel S3. The typical Root Mean Square (RMS) roughness is given in Table 1 for each group of microchannels. One can remark that the roughness of the microchannel A4 is not equal to that of the other microchannels of this group, because the microchannel A4 was fabricated separately, not at the same fabrication conditions as the other microchannels. However, it was maintained in the group A because its roughness remains close to those of the other microchannels of that group.

\subsection{Mass Flow Rate Measurement Technique}

The mass flow rate though a microchannel can be calculated from the equation of state for an ideal gas

$$
p V=M \mathcal{R} T,
$$

by assuming steady flow conditions. Here $V$ is the volume, $p$ and $T$ are, respectively, the pressure and temperature of a gas, $M$ is the mass of a gas and $\mathcal{R}$ is the specific gas constant. Using the constant volume technique, the mass flow rate can be derived from Eq. (1) as it was shown in [21] and [15]

$$
\dot{M}=\frac{d M}{d t}=\frac{V}{\mathcal{R} T} \frac{d p}{d t}(1-\theta), \quad \text { with } \quad \theta=\frac{d T / T}{d p / p} .
$$

In above equation, $\theta$ represents the ratio of fractional change of the temperature to the fractional change of pressure. If $\theta$ is small enough compared to 1 then it can be neglected. Below we estimate the order of $\theta$ in our experimental conditions.

In this work, we consider an isothermal flow through a microchannel connecting two large tanks maintained at pressures $p_{\text {in }}$ and $p_{\text {out }}$, which remain close to constant values. The pressure variations in each tank during a measurement are less than $1 \%$, thus the flow may be considered as a steady flow. Consequently, the relative pressure variation $d p / p$ in a tank is of the order of $10^{-2}$. The relative variation of the temperature $d T / T$ is calculated from the standard 
deviation $(d T / T=s)$

$$
s=\sqrt{\frac{1}{n-1} \sum_{i=1}^{n}\left(T_{i}-\bar{T}\right)^{2}},
$$

where $\bar{T}$ is the average temperature during the experimental time, $T_{i}$ is the instantaneous temperature measurement and $n$ is the number of measurements during the experimental time. This standard deviation $s$ is smaller than $2 \times 10^{-4}$ for all the experiment series, so $d T / T$ is of the order of $10^{-4}$. $\theta$ in Eq.(2) is clearly less than $2 \times 10^{-2}$, so this term can be neglected.

Thus, from Eq. (2) the mass flow rate $\dot{M}^{\text {exp }}$ expression can be simplified as

$$
\dot{M}^{\exp }=\frac{V}{\mathcal{R} T} \frac{d p}{d t} .
$$

To calculate the mass flow rate using Eq. (4), the registered data of the pressure $p_{i}$ at different time instants $t_{i}$ are used. The stationary assumption can justify physically the implementation of first order polynomial expression in $t_{i}$

$$
p\left(t_{i}\right)=a t_{i}+b,
$$

where the slope $a$ of the function $p\left(t_{i}\right)$ is equal to $d p / d t$.

The global uncertainty on the mass flow rate measurements is around $5 \%$. This includes the uncertainty of the volume measurements, which is of the order of $2 \%$. The error on the coefficient $a$ determination, Eq. (5), is of the order of $1 \%$. The relative error related to the neglecting of term $\theta$ in Eq. (2) is of the order of $2 \%$ and it is included also in the global uncertainty estimation of the mass flow rate measurements.

\section{Analytical Method in the Slip Regime}

In this section we present analytical results related to the influence of the lateral wall on gas flow in rectangular cross-section microchannels. In the following we continue to use the same notations, as those already introduced in Section 2.2, namely, we denote the channel height $h$, the channel width $w$ and the channel length $L$, see Fig. 3. The main results are presented in Ref. [17]. We recall here only some important parts that are indispensable to go forward with the new analytical calculations that will permit to take into account the different properties of the channel walls.

\subsection{Governing Equations and Slip Boundary Conditions}

As it is well known, for an isothermal gas flow through long microchannel, the system of the Navier-Stokes (NS) equations may be simplified at the zero order of the small channel aspect ratio parameter $\varepsilon_{h L}=h / L \ll 1$. Then, the momentum conservation equation appears under the form of the Stokes equation as

$$
\frac{\partial^{2} u_{z}}{\partial x^{2}}+\frac{\partial^{2} u_{z}}{\partial y^{2}}=\frac{1}{\mu} \frac{d p}{d z}
$$


Here $u_{z}$ is the gas velocity along the $z$ axis, and $x, y$ are the directions that correspond to the channel width and height, respectively, see Fig. 3. The gas viscosity $\mu$ in Eq. (6) is calculated in the frame of the Variable Hard Sphere (VHS) model [22] as $\mu=\mu_{\text {ref }}\left(T / T_{r e f}\right)^{\omega}$, where $\omega$ is the viscosity index, which depends on the gas nature, $\mu_{r e f}$ is the reference viscosity at the reference temperature $T_{r e f}$.

As mentioned previously, we consider here the slip flow regime characterized by a Knudsen number varying in the range $0.01<K n<0.3$. The Knudsen number, $K n=\lambda / h$, is defined as the ratio of the molecular mean free path $\lambda$ to the characteristic channel cross-section dimension $h$, see Fig. $3(\mathrm{~b})$. As it is well known, in the slip regime, the Stokes equation (6) must be supplemented with slip boundary conditions. Moreover, it is important to remember that, since we have an axis (or a plane) of symmetry, the second order terms with respect to the Knudsen number are negligible in the conservation equation system and they are important only when they result from the slip boundary condition [23]. Therefore, in the geometry considered here, according to the results given in Ref. [23], a pertinent slip velocity boundary condition may be written at the second order following $\lambda$ as

$$
\left.u_{z}\right|_{s}= \pm\left. A_{1} \lambda \frac{\partial u_{z}}{\partial \vec{n}}\right|_{s}-\left.A_{2} \lambda^{2} \Delta u_{z}\right|_{s},
$$

where the mean free path $\lambda$ is calculated using the VHS model [22] as

$$
\lambda=k_{\lambda} \frac{\mu}{p} \sqrt{2 \mathcal{R} T}, \quad k_{\lambda}=\frac{(7-2 \omega)(5-2 \omega)}{15 \sqrt{\pi}} .
$$

In Eq. (7) the subscript "s" corresponds to the quantities, calculated at the surface, $\vec{n}$ represents the normal to the surface, and $A_{1}$ and $A_{2}$ are the coefficients depending on the gas-surface interaction. Moreover, in a more recent study, it was shown [24] that the Knudsen layer influence does not modify the form of Eq. (7), only the analytical form of $A_{1}$ and $A_{2}$ coefficient becomes different, when the contribution of the Knudsen layer is taken into account [24].

Now we will resolve Eq. (6) subjected to boundary condition, Eq. (7). It results from Eq. (6) that $\Delta u_{z}$ does not depend on the $x$ and $y$ coordinates whatever the point considered in the channel cross-section. Thus, $\left.\Delta u_{z}\right|_{s}$ in Eq. (7) is constant at any point of the section perimeter. The same comment may be applied to the molecular mean free path $\lambda$, due to the negligent variation of pressure in the cross section. Then, it is convenient, first from a calculation simplification point of view, to use the function change

$$
u^{*}=u_{z}+\left.A_{2} \lambda^{2} \Delta u_{z}\right|_{s} .
$$

Applying this change does not change the form of Eq. (6) and the system, Eqs. (6) and (7), can be rewritten using $u^{*}$ as

$$
\Delta u^{*}=\frac{1}{\mu} \frac{d p}{d z}
$$




$$
\left.u^{*}\right|_{s}= \pm\left. A_{1} \lambda \frac{\partial u^{*}}{\partial n}\right|_{s}
$$

Using the symmetry planes of the problem $(x \geq 0$ and $y \geq 0)$, the boundary conditions (11) in a quarter of the channel cross-section may be detailed as following:

$$
\left\{\begin{array}{l}
\frac{\partial u^{*}}{\partial x}(0, y)=0 \\
u^{*}(w / 2, y)=-A_{1} \lambda \frac{\partial u^{*}}{\partial x}(w / 2, y)
\end{array}\right.
$$

and

$$
\left\{\begin{array}{l}
\frac{\partial u^{*}}{\partial y}(x, 0)=0, \\
u^{*}(x, h / 2)=-A_{1} \lambda \frac{\partial u^{*}}{\partial y}(x, h / 2) .
\end{array}\right.
$$

\subsection{Basic Solving Methodology}

The present approach is mainly based on the spectral properties of the partial Laplace operators included in the Stokes equation (10). When applied to the vector space of the $L_{2}$ functions, defined on a finite spatial domain $D$ (here the channel cross-section), the Laplacian (or the partial Laplacian) is characterized by an eigen-function discrete spectrum.

Expanding the solution $u^{*}$ of Eq. (10) on the eigen functions $f_{n}(y)$ of the partial Laplacian $\frac{\partial^{2}}{\partial y^{2}}$, where functions $f_{n}(y)$ verify border conditions of cancellations, similar to Eq. (13), we change Eq. (10) into a set of differential equations easy to solve. Indeed the $f_{n}(y)$ set provides an orthogonal basis of the space $L_{2}(y)$ of the $y$ functions, defined on the channel cross-section and verifying, at the limits, conditions similar to Eq. (13). Moreover, the eigenvalues associated with $f_{n}(y)$ form a discrete countable series of real negative numbers $-\nu_{n}^{2}$. Thus, the solution $u^{*}$ has the form

$$
u^{*}=\sum_{n=0}^{\infty} g_{n}(x) f_{n}(y),
$$

where $n$ is a natural integer. Taking into account the properties of $f_{n}$ functions, we find

$$
f_{n}(y)=a_{n} \cos \left(\nu_{n} y\right)
$$

$$
\operatorname{cotan}\left(\beta_{n}\right)=2 A_{1} K n \beta_{n}
$$

$$
\beta_{n}=\nu_{n} h / 2 .
$$

The $g_{n}(x)$ functions in Eq. (14) have to be calculated in next Section to find the solution $u^{*}$. 


\subsection{Search for $g_{n}(x)$ and for the reduced velocity}

Now we will obtain the explicit expression for $g_{n}(x)$ functions involved in Eq.(14). The second member of Eq. (10) depends only on the $z$ space variable and may be expanded on the $f_{n}(y)$ basis functions. Then, from Eq. (10) and using $u^{*}$ velocity expansion in form of Eq. (14), we obtain a linear second order differential equation giving $g_{n}(x)$. Finally, solving this equation and taking also into account Eqs. (12), (15), (16) and (17) we deduce

$$
u^{*}=\frac{h^{2}}{\mu} \frac{d p}{d z} \sum_{n=0}^{\infty} \phi_{n}(x, y)
$$

where

$$
\phi_{n}(x, y)=\frac{\sin \left(\beta_{n}\right) \cos \left(2 \beta_{n} y / h\right)}{\beta_{n}^{2}\left(2 \beta_{n}+\sin \left(2 \beta_{n}\right)\right)}\left(\frac{\cosh \left(2 \beta_{n} x / h\right)}{\cosh \left(\beta_{n} w / h\right)+\beta_{n} \varepsilon \sinh \left(\beta_{n} w / h\right)}-1\right) .
$$

In the previous expression, the small parameter $\varepsilon$ is defined as

$$
\varepsilon=2 A_{1} K n, \quad \varepsilon \ll 1 .
$$

Let us remind that in Ref. [17] we explained why, when transforming Eq. (6) into Eq. (10), the precision of the results given by the NS equations is not physically modified, and, more practically, why resolving first the system, Eqs. (10) and (11), and using in fine Eq. (9) to attain $u_{z}$ we obtain strictly the same physical result as by solving the system, Eqs. (6) and (7). Hereafter, we apply the correlative remark of Ref. [17] explaining that in $u^{*}$ expression only the first order terms following the Knudsen number are physically significant. Therefore, expression (18) of $u^{*}$ is expanded up to the first order according to the small parameter $\varepsilon$, defined in (20) as proportional to the Knudsen number. Precisely

$$
u^{*}=u_{0}^{*}+\varepsilon\left(\frac{d u^{*}}{d \varepsilon}\right)_{0},
$$

where

$$
u_{0}^{*}=\left.u^{*}\right|_{\varepsilon=0}, \quad\left(\frac{d u^{*}}{d \varepsilon}\right)_{0}=\left.\frac{d u^{*}}{d \varepsilon}\right|_{\varepsilon=0} .
$$

Since $u^{*}$ depends on $\varepsilon$ not only explicitly through relation (20), but also implicitly via $\beta_{n}$ related with the local Knudsen number through Eq. (16); then by using Eq. (20), equation (16) is now written formally as:

$$
F\left(\beta_{n}, \varepsilon\right)=\operatorname{cotan}\left(\beta_{n}\right)-\varepsilon \beta_{n}=0 .
$$

Therefore, the calculation of the total derivative of $u^{*}$ must be written as:

$$
\left(\frac{d u^{*}}{d \varepsilon}\right)_{0}=\sum_{n=0}^{\infty}\left(\frac{\partial u^{*}}{\partial \beta_{n}} \frac{d \beta_{n}}{d \varepsilon}\right)_{0}+\left(\frac{\partial u^{*}}{\partial \varepsilon}\right)_{0}
$$


The calculations of $u_{0}^{*}$, and the derivatives $\left(\frac{\partial u^{*}}{\partial \beta_{n}}\right)_{0}$ and $\left(\frac{\partial u^{*}}{d \varepsilon}\right)_{0}$ in Eq. (24) have been easily done by using successively Eqs. (18) and (19), and the following results deduced from Eq. (23):

$$
\left(\beta_{n}\right)_{0}=\frac{\pi}{2}(2 n+1)
$$

In addition, the calculation of expression $\left(\frac{d \beta_{n}}{d \varepsilon}\right)_{0}$ requires to use again Eq. (23), where $F$ appears as an implicit function of $\beta_{n}$ and $\varepsilon$. Expressing now the partial derivative of $F$ one obtains

$$
\left(\frac{d \beta_{n}}{d \varepsilon}\right)=-\frac{\partial F / \varepsilon}{\partial F / \beta_{n}}=-\frac{\beta_{n}}{1+\operatorname{cotan}^{2}\left(\beta_{n}\right)+\varepsilon} .
$$

To calculate $\left(\frac{d \beta_{n}}{d \varepsilon}\right)_{0}$ from the second member of Eq. (26), we have to take $\varepsilon=0$ and $\left(\beta_{n}\right)_{0}$ from Eq. $(25)$, which leads to

$$
\left(\frac{d \beta_{n}}{d \varepsilon}\right)_{0}=-\frac{\pi}{2}(2 n+1)
$$

Then, we finally obtain following expression for velocity

$$
u^{*}=\frac{h^{2}}{\mu} \frac{d p}{d z}\left(\sum_{n=0}^{\infty}\left[\phi_{n}\right]_{0}+\varepsilon \sum_{n=0}^{\infty}\left(\left[\varphi_{n}\right]_{0}+\left[\chi_{n}\right]_{0}\right)\right),
$$

with $\left(\phi_{n}\right)_{0},\left(\varphi_{n}\right)_{0}$ and $\left(\chi_{n}\right)_{0}$ given in Ref. [17], where the velocity $u_{z}$ is deduced from Eq. (A 15).

\subsection{Mass Flow Rate}

Considering now the mass flow rate defined as

$$
\dot{M}=\frac{p}{\mathcal{R} T} \int_{-w / 2}^{w / 2} \int_{-h / 2}^{h / 2} u_{z}(x, y) d x d y
$$

we obtain new mass flow rate expression

$$
\dot{M}=\dot{M}_{P}\left(1+6 A_{1} \frac{T_{n}}{S_{n}} K n_{m}+\frac{A_{2} \pi^{4}}{16 S_{n}} \frac{\mathcal{P}+1}{\mathcal{P}-1} \ln \mathcal{P} K n_{m}^{2}\right),
$$

where $\dot{M}_{P}$ is the analytical expression of the Poiseuille mass flow rate in the hydrodynamic flow regime

$$
\dot{M}_{P}=\frac{h^{3} w \Delta p p_{m}}{12 \mu \mathcal{R} T L} V_{n}
$$

In Eq. (30) $K n_{m}$ is the mean Knudsen number, based on the mean pressure $p_{m}=0.5\left(p_{\text {in }}+p_{\text {out }}\right)$ and on the characteristic length $h, \Delta p=p_{\text {in }}-p_{\text {out }}$, $\mathcal{P}=p_{\text {in }} / p_{\text {out }}$,

$$
S_{n}=\frac{\pi^{4}}{96}-\frac{2 h}{\pi w} S_{1}, \quad \text { with } \quad S_{1}=\sum_{n=0}^{\infty} \frac{\tanh (0.5 \pi(2 n+1) w / h)}{(2 n+1)^{5}}
$$




$$
V_{n}=\frac{96}{\pi^{4}} S_{n}
$$

and

$$
T_{n}=\frac{4}{3} S_{n}-\frac{1}{3}\left(1-\frac{h}{w}\right) S_{3}, \quad S_{3}=\sum_{n=0}^{\infty} \frac{\tanh ^{2}(0.5 \pi(2 n+1) w / h)}{(2 n+1)^{4}} .
$$

\subsection{Extension to Two Surface Materials}

It is possible to extend the method, briefly presented above and published in Ref. [17], to the case when the walls of the channel are fabricated differently, see comments in Section 2.2. But, unfortunately, it is then necessary to restrict the new approach to the first order according to the Knudsen number. Indeed, in this case, the second order term of the boundary condition, Eq. (7), does not involve the Laplacian form of constant value in the channel cross-section. Remember, that the presence of this term allowed us to make the function change written in Eq. (9).

Thus, we consider hereafter, at the first order only, the case of a channel, where both vertical channel walls are made from a first material corresponding to a first order slip coefficient, $A_{1 w}$, and both horizontal walls are made from another material, characterized with the slip coefficient $A_{1 h}$. In the following, to avoid cumbersome expressions we use simplified notations $A_{w}$ and $A_{h}$ for the slip coefficients related to the vertical and horizontal walls, respectively. The boundary conditions on the wall reduce now to an equation similar to Eq. (11), where $u^{*}$ is now changed into $u_{z}$ and where the slip coefficient changes following the wall orientation. Of course, the Stokes equation directly solved here is Eq. (6) involving $u_{z}$ instead of Eq. (10) involving $u^{*}$. Detailing now the boundary condition in the $(x, y)$ coordinates we obtain equations similar to Eqs. (12) and (13). Again, $u^{*}$ is changed into $u_{z}$ and $A_{1}$ is successively changed into $A_{w}$ and $A_{h}$ which were respectively related to two dimensionless numbers, small compared to one and associated to the Knudsen number:

$$
\varepsilon_{w}=A_{w} K n, \quad \varepsilon_{h}=A_{h} K n .
$$

Consequently, Eqs. (14) - (17) are maintained, nothing however that the coefficient $A_{1}$ is here changed into $A_{h}$ because deriving from the new forms of Eqs. (13) and (23), namely:

$$
F\left(\beta_{n}, \varepsilon_{h}\right)=\operatorname{cotan}\left(\beta_{n}\right)-\varepsilon_{h} \beta_{n}=0 .
$$

Then, the search of $u^{*}$ is now replaced by the search of $u_{z}$. We use practically the same equations, but as previously noticed, in Eq.(12), $A_{1}$ is changed into $A_{w}$. Consequently, in the new form of Eq. (19) only $\varepsilon_{w}$ would appear explicitly, replacing $\varepsilon$. Thus, the $u_{z}$ expansion takes the form

$$
u_{z}=\left(u_{z}\right)_{0}+\varepsilon_{w}\left(\frac{d u_{z}}{d \varepsilon_{w}}\right)_{0} .
$$


However, in expression $\left(\frac{d u_{z}}{d \varepsilon_{w}}\right)_{0}$ the parameter $\beta_{n}$ is involved, but $\beta_{n}$ is calculated from Eq. (36) and so related to $\varepsilon_{h}$, thus we obtain

$$
\left(\frac{d u_{z}}{d \varepsilon_{w}}\right)_{0}=\sum_{n=0}^{\infty}\left(\frac{\partial u_{z}}{\partial \beta_{n}} \frac{d \beta_{n}}{d \varepsilon_{h}} \frac{d \varepsilon_{h}}{d \varepsilon_{w}}\right)_{0}+\varepsilon_{w}\left(\frac{\partial u_{z}}{\partial \varepsilon_{w}}\right)_{0}
$$

In previous expression the derivative $\frac{d \beta_{n}}{d \varepsilon_{h}}$ is obtained from Eq. (36) in the same way as in Eq. (26), and where $\frac{d \beta_{n}}{d \varepsilon_{w}}=\frac{d \beta_{n}}{d \varepsilon_{h}} \frac{d \varepsilon_{h}}{d \varepsilon_{w}}$ with $d \varepsilon_{h} / d \varepsilon_{w}=A_{h} / A_{w}$. Finally, we obtain an expression close to Eq. (28), where $\varepsilon$ is changed into $\varepsilon_{w}$ and where appears the ratio $A_{h} / A_{w}$ coming from the new derivative $d \beta_{n} / d \varepsilon_{w}$ :

$$
u_{z}=\frac{h^{2}}{\mu} \frac{d p}{d z}\left(\sum_{n=0}^{\infty}\left(\phi_{n}\right)_{0}+\varepsilon_{w} \sum_{n=0}^{\infty}\left[\left(\frac{A_{h}}{A_{w}} \varphi_{n}\right)_{0}+\left(\chi_{n}\right)_{0}\right]\right) .
$$

Naturally the functions $\phi_{n}, \varphi_{n}, \chi_{n}$ are exactly the same as the corresponding quantities given in [17]. To provide the complete analytical development these functions are also given in Appendix A.

Then, calculating the mass flow rate, we find expressions close to those presented in [17]. Considering the group of the equations, allowing the calculation of the mass flow rate, Eqs. (30) - (34), the second order term vanish, and $A_{1}$ becomes $A_{w}$, so new mass flow rate expression reads formally:

$$
\dot{M}=\dot{M}_{P}\left(1+6 \frac{A_{w}}{S_{n}} T_{n}\left(A_{w}, A_{h}\right) K n_{m}\right) .
$$

Then, parameter $T_{n}\left(A_{w}, A_{h}\right)$ in Eq. (34), is slightly modified and it becomes:

$$
T_{n}\left(A_{w}, A_{h}\right)=\frac{4}{3} \frac{A_{h}}{A_{w}} S_{n}-\frac{1}{3}\left(\frac{A_{h}}{A_{w}}-\frac{h}{w}\right) S_{3},
$$

where $S_{3}$ is determined by Eq. (34). The other quantities remain exactly the same as previously.

\section{Implementation of the Methodology}

The two analytical methods, discussed in Section 3, which are based on two different assumptions, are applied hereafter in order to model the gas flow through rectangular channel, taking into account the effect of its twodimensional cross-section area.

Following the first method, we suppose that for each channel all the walls (vertical and horizontal) are characterized by the same material and the same roughness. In this method we use a second order approach: for each channel we determine a global coefficient $A_{1}$ at the first order and another global coefficient $A_{2}$ at the second order according to the Knudsen number.

The second method is based on a different assumption. In this approach, we suppose that the etching process used to fabricate the channels can induce 
a roughness difference between the vertical and horizontal walls. In this case, we use a first order approach, which leads to two first order coefficients to be determined, $A_{w}$ and $A_{h}$, for the vertical and horizontal walls, respectively. But to find these two coefficients we need an additional hypothesis, namely, in each group of channels (A, E and $\mathrm{S}$ ) all the vertical walls are considered similar, i.e. characterized with a same $A_{w}$, while all the horizontal walls are supposed to have the same $A_{h}$ coefficient. Finally, considering the experimental values of the mass flow rate, we calculate the coefficients, following each method described above, then, we determine in each case what is the more pertinent method to retain.

\subsection{Slip coefficient extraction with a single surface material method}

It is assumed here that the horizontal and vertical walls material is the same for each channel. The analytical expression for the mass flow rate, obtained in Section 3.4, see Eq. (30), is used here to extract the "experimental" slip coefficient. As it was obtained from the theoretical analysis, see Section 3.4, the analytical mass flow rate can be expressed in the second order polynomial form according to the Knudsen number. By dividing the dimensional mass flow rate, Eq. (30), by the analytical expression of the Poiseuille mass flow rate in the hydrodynamic flow regime, $\dot{M}_{P}$, Eq. (31), we obtain the following dimensionless analytical expression:

$$
S^{T}=B_{0}^{T}+B_{1}^{T} K n_{m}+B_{2}^{T} K n_{m}^{2},
$$

where

$$
B_{0}^{T}=1, \quad B_{1}^{T}=6 A_{1} \frac{T_{n}}{S_{n}}, \quad \text { and } \quad B_{2}^{T}=\frac{A_{2} \pi^{4}}{16 S_{n}} \frac{\mathcal{P}+1}{\mathcal{P}-1} \ln \mathcal{P} .
$$

We can also assume that the measured values of the mass flow rate can be represented by an analogous dimensionless second order polynomial form according to the Knudsen number:

$$
S_{f}^{e x p}=B_{0}^{e x p}+B_{1}^{e x p} K n_{m}+B_{2}^{e x p} K n_{m}^{2} .
$$

Comparing expressions (42), (43) and (44), the coefficients $A_{1}$ and $A_{2}$ are expressed as

$$
A_{1}=\frac{\sigma_{p}}{k_{\lambda}}=\frac{B_{1}^{e x p} S_{n}}{6 T_{n}}, \quad \text { and } \quad A_{2}=\frac{\sigma_{2 p}}{k_{\lambda}^{2}}=\frac{16 B_{2}^{e x p} S_{n}}{\pi^{2} \ln \mathcal{P}} \frac{\mathcal{P}-1}{\mathcal{P}+1} .
$$

Therefore, the "experimental" slip coefficients of first and second order, $\sigma_{p}$ and $\sigma_{2 p}$, respectively, are found as

$$
\sigma_{p}=\frac{B_{1}^{e x p} k_{\lambda} S_{n}}{6 T_{n}}, \quad \text { and } \quad \sigma_{2 p}=\frac{16 B_{2}^{e x p} k_{\lambda}^{2} S_{n}}{\pi^{2} \ln \mathcal{P}} \frac{\mathcal{P}-1}{\mathcal{P}+1} .
$$

It is clear from the previous expressions that the lateral wall influence is present in both coefficients $B_{1}^{e x p}$ and $B_{2}^{e x p}$ through the coefficients $S_{n}$ and $T_{n}$, see Eqs. 
(32) - (34). The dependence of velocity slip coefficients, $\sigma_{p}$ and $\sigma_{2 p}$, on the molecular interaction model, here VHS model [22], comes from the coefficient $k_{\lambda}$, see Eqs. (8), (46).

By solving numerically the linearized BGK kinetic equation and using the Maxwellian diffuse-specular scattering kernel for various values of the accommodation coefficient $\alpha$, the authors of [25] have proposed the relation between the first order slip coefficient $\sigma_{p}$ and the accommodation coefficient $\alpha$. This expression was further improved in [26] and it becomes:

$$
\sigma_{p}(\alpha)=\frac{2-\alpha}{\alpha}\left(\sigma_{p}(1)-0.1211(1-\alpha)\right)
$$

where $\sigma_{p}(1)=1.016$ is the velocity slip coefficient for $\alpha=1$ calculated in [27].

For all gases the second order treatment appeared us the most pertinent in slip regime using the same criteria as those used in [15]. The values of the main parameters used as criteria appear in Tables 8, 10 and 12. For each Knudsen number range we calculated the determination coefficient, $r^{2}$, the squared residual sum $s_{r}=\sqrt{\left(1 /(n-p) \sum_{i=1}^{n} e_{i}^{2}\right.}$, and the standard error on the fit curves $E_{s}=\sqrt{1 / n \sum_{i=1}^{n} e_{i}^{2}} / \overline{S^{e x p}}$, where $e_{i}=S_{i}^{\text {exp }}-S_{f_{i}}^{\text {exp }}$ is the local difference between the measured and fitting values and it represents the local fitting error, $n$ is the number of measurements, $p$ is the number of the unknown coefficients of the fitting model, and $\overline{S^{e x p}}=1 / n \sum_{i=1}^{n} S_{i}^{e x p}$ is the averaged value of the measured values. Analyzing the values of these coefficients given in Tables 8, 10 and 12, respectively, for first and second order approaches, we found that the determination coefficient of second order is slightly more closer to one than that of first order. However, the squared residual sum and the standard error are of the same order for the both fitting. Therefore, we can conclude that for the studied cases both first and second fittings reproduce well the measurements.

\subsection{Slip coefficient extraction with two surface materials method}

It is assumed here that for each channel the horizontal and vertical walls roughnesses are different. By analogy with the previous methodology we can express the analytical mass flow rate, Eq. (40), in the following dimensionless first order polynomial form, by dividing expression (40) by $\dot{M}_{P}$ :

$$
S^{T}=C_{0}^{T}+C_{1}^{T} K n_{m},
$$

where

$$
C_{0}^{T}=1, \quad C_{1}^{T}=6 A_{w} \frac{T_{n}\left(A_{w}, A_{h}\right)}{S_{n}},
$$

here $T_{n}\left(A_{w}, A_{h}\right)$ and $S_{n}$ are given by Eqs. (41) and (32), respectively. We assume also here that the measured values of the mass flow rate can be presented using a first order dimensionless polynomial form:

$$
S_{f}^{e x p}=C_{0}^{e x p}+C_{1}^{e x p} K n_{m} .
$$


Then, by comparing expressions (48) and (50) we obtain:

$$
A_{w} T_{n}\left(A_{w}, A_{h}\right)=\frac{1}{6} C_{1}^{e x p} S_{n}
$$

However, practically we cannot calculate both values of $A_{w}$ and $A_{h}$ simultaneously, because we have only one equation (51) to determine them. Therefore, as it was mentioned previously, we will consider that all the channels belonging to the same group have the same coefficients $A_{w}$ and $A_{h}$. Doing so, we can obtain, for each group of channels sufficient quantity of data to extract these two coefficients.

Practically we consider the following approach. The theoretical expression of the dimensionless mass flow rate, Eqs. (48), (49), can be written for a channel by equalizing it to the experimental expression, $S^{\exp }$, as following:

$$
A_{0}+A_{h}\left(8-S_{0}\right) K n_{m}+A_{w} \frac{h}{w} S_{0} K n_{m}=S^{e x p}, \quad S_{0}=2 \frac{S_{3}}{S_{n}} .
$$

The previous expression is then written for all measured values of the dimensionless mass flow rates $S^{\exp }$ and for all channels in each group. We write so a large number of equations involving the same unknown coefficients $A_{0}, A_{w}$ and $A_{h}$. Here $A_{0}$ coefficient is a free parameter, which theoretically has to be equal to 1 . However, we decided to calculate its value from the fitting procedure (multiple linear regression), as we have done for two other coefficients, $A_{w}$ and $A_{h}$. We obtain here an overdetermined system of equations taking a matrix form, involving a vector of unknown coefficients, where each line corresponds to a measurement. This system is classically resolved by minimizing the norm of its residual vector, where the coefficients $A_{0}, A_{w}$ and $A_{h}$ are included, then, their convenient values are extracted.

\section{Results and Discussion}

The mass flow rates through all the microchannels considered in this study were measured for three gases, Helium, Nitrogen and Argon. Then, we extract the velocity slip and the accommodation coefficients from the mass flow rate measured for the series of channels type $A, E$ and $S$ by considering first the same roughness on the vertical and horizontal walls, Section 5.1, and then, by considering the second approach, Section 5.2, which allows us to consider different roughnesses on the vertical and horizontal walls.

The geometrical characteristics for the channels of group A are provided in Table 1. The experimental conditions for the microchannels of this group are summarized in Tables 2 and 3. To accomplish one of the goals fixed in this article, namely the study of the surface roughness influence on the momentum transfer between a gas and a surface, we conducted an additional series of mass flow rate measurements through the microchannels of group $E$ which have similar rectangular cross-section aspect ratios and the same material $(A u)$ of the internal surfaces coating as the microchannels of group $A$. However, 
the microchannels of group $\mathrm{E}$ have a surface roughness 12 times greater than that of the microchannels $A$, see Table 1 . The experimental conditions for the microchannels of group $E$ are summarized in Tables 4 and 5 .

To study the influence of the surface nature on the momentum transfer between a gas and a surface, the microchannels of the group $S$ were fabricated by coating the internal surfaces with a layer of silica $\left(\mathrm{SiO}_{2}\right)$. The channel of this group were etched in a wafer to a depth $h$, than covered by an other plate wafer. This technology was different from the fabrication of $A$ and $E$ channels, where two half parts of depth $(h / 2)$ were etched in a wafer. However, the surface roughness of group $S$ channels is similar to that of microchannels of the group $A$. Therefore, a comparison of the TMAC results between these two groups can clarify the influence of the surface materials on the momentum exchange between a gas and a surface. The experimental conditions for microchannels of the group $S$ are given in Tables 6 and 7 .

\subsection{Results for the single surface material method}

In this Section we present the results of the first method, where the horizontal and vertical walls of each channel are considered to have the same properties (material and roughness). The experimental data of the mass flow rate in two Knudsen number ranges: $[0,0.1]$ and $[0,0.3]$ are fitted using the first and second order polynomial forms, respectively, see Section 4.1. Then, the first order slip coefficient in the case of the first order fit, and first and second order coefficients in the case of the second order fit, are extracted. Finally, the tangential momentum accommodation coefficients are calculated for each channel. All data of the experimental fitting coefficient and the slip and tangential momentum accommodation coefficients, are provided in Tables 8 - 13. Examples of the first and second order polynomial fits of the experimental data are provided on Figures $4-6$. By analyzing these data, we will conclude on the validity of the proposed methodology, as well as on the influence of the surface nature and the surface roughness on the gas-surface interaction.

\subsubsection{Influence of the channel cross-section aspect ratio}

The influence of the microchannel aspect ratio on the gas flow through microchannels is assumed to be taken into account by the factors $S_{n}, T_{n}$ and $V_{n}$ in the expression for the mass flow rate, see Eqs. (30) - (34). Considering the results, reported in Tables 8, 10 and 12, we found that the values of the fitting coefficients, $B_{0}^{\exp }$ for all groups of channels are close to the theoretical value $B_{0}^{T}=1$, Eqs. (30) and (43). If we check carefully the values of the coefficient $B_{0}^{e x p}$ for the $S$ and $A$ channels, they are different from 1 by $1.6 \%$ and $2.7 \%$, respectively. However, for the channels of group $E$ the difference increases to $6.6 \%$, see Table 10. The detailed analysis of the possible nature of this discrepancy, see Appendix B, allows us to conclude that the probable factor influencing the results of the microchannels $E$ can be the gold layer takeoff inside the microchannels, which causes the change of their cross-section shape.

The fact that the fitting coefficients $B_{0}^{e x p}$ remains close to 1 , when the channel aspect ratio changes, confirms the correctness of the theoretical expression 
of the mass flow rate, Eq. (30). In addition, by analyzing Tables 8, 10 and 12, it can be seen that for each group of channels and for each gas, the extracted coefficients (first and second order velocity slip and accommodation coefficients) have very close values, regardless the channel cross-section changes.

Furthermore, in our bi-dimensional modeling, we supposed that all channel walls are homogeneous and that the gas-surface interaction is the same for the upper, bottom and lateral walls. Therefore, when a remaining influence of the cross-section aspect ratio appears in the behavior of the first and second order velocity slip coefficients, it may be explained by the difference of the gas-surface interaction between the vertical and horizontal walls or by the deformation of the cross-section shape.

\subsubsection{Influence of the surface nature}

We are going to focus our analysis on the microchannels of groups $A$ and $S$. The wall roughness of the channels in these groups is very low, of the order of one nanometer and the aspect ratio ranges are comparable, see Table 1. It is clear that practically only the nature of the walls is different between these groups of microchannels. The microchannels of group $A$ are covered with gold $(A u)$ and the microchannels of group $S$ are covered with Silica $\left(\mathrm{SiO}_{2}\right)$. For all channels of group $A$, and for all the used gases, the tangential momentum accommodation coefficients are found to be very close, each other, except for microchannel $A 1$, which has an accommodation coefficient smaller than the other microchannels of this group, see Table 9.

For the microchannels of group $S$, see Figure 7, all the tangential momentum accommodation coefficients are very similar and their values are around 0.92 . These small deviations of the tangential momentum accommodation coefficient from its mean value are most of the time of the order of the uncertainty of measurement, see Table 13. Therefore, in this case the TMAC seems to be weakly dependent on the gas nature.

However, if comparing the TMAC values, obtained for all $S$ group microchannels with those obtained for the microchannels $A 2, A 3$ and $A 4$, one can notice that lower values are globally obtained for the microchannels of group $S$. As previously noted, both groups of microchannels $A$ and $S$ have almost the same surface roughness, therefore, from this comparison it can be concluded that the gold $(\mathrm{Au})$ material is slightly more diffusive than the silica $\left(\mathrm{SiO}_{2}\right)$.

\subsubsection{Influence of the surface roughness}

The microchannels of both groups $(A$ and $E$ ) differ only by roughness of their internal surfaces, see Table 1 . The other characteristics, like the surface material, or the gas species involved in this analysis, are the same. The respective cross-section aspect ratios are also similar. Therefore, it would be very interesting to compare directly the influence of the surface roughness through the respective slip and accommodation coefficients, obtained for the channels of groups $A$ and $E$. But, unfortunately, as it is explained in Appendix B, a probable gold layer takeoff inside the microchannels $E$ modified the cross sections and the flows, which made them unsuitable for the extraction of both exact 
coefficients in the frame of the models developed in this work. Nevertheless, qualitative and comparative descriptions are possible notably concerning the different gases behaviors. The analysis is focused here on the dependence of $\sigma_{p}$ and $\alpha$ coefficients on the molecular mass of the gases.

From Table 11 one can observe for microchannels $E$ that higher is the molecular mass smaller is the accommodation coefficient, even for diatomic gas like $N_{2}$. However, in some cases, this hierarchy may be perturbed due to the influence of the internal energy of the polyatomic gas molecules. Conversely, in the case of groups $A$ and $S$, we have mentioned in the previous paragraphs that for the microchannels of these groups, the TMAC dependence on the gas nature, hence on its molecular mass, is not significant, almost null, for several cases, see Tables 9 and 13.

Comparing the gases behavior obtained in three groups of channels, one can conclude that the wall roughness plays an important role on the TMAC dependence on the molecular mass. First, the smooth surfaces of microchannels $A$ and $S$ make the influence of the gas type, i.e. of the gas molecular mass, on the TMAC almost nonexistent. Then, when the surface roughness increases, the gas molecules stand out by their molecular mass, such as the highest TMAC value is obtained for the lightest gas, at least for the monoatomic molecules. Finally, the influence of the roughness on the value of the accommodation coefficient itself will be discussed below, in the global frame of two theoretical approaches.

\subsubsection{Second order velocity slip coefficient}

When the experimental data are fitted using the second order polynomial form, Eq. (44), two velocity slip coefficients, $\sigma_{p}$ and $\sigma_{2 p}$, are extracted using expressions (45) and (46). The values of both coefficients are presented in Tables 9,11 and 13. Most of $\sigma_{2 p}$ values lie in the range from 0.15 to 0.25 . However, for the microchannels with small aspect ratios, smaller and even negative values of $\sigma_{2 p}$ coefficient are obtained, see Tables 9, 11 and 13 .

The discussion about the physical significance of the second order velocity slip coefficient and its theoretical values can be found in Refs. [28], [23], [29], [24], where the values of $\sigma_{2 p}$ coefficient were found to be equal to $0.884,0.766$, 0.243 and 0.184 , respectively. The measured here values of the second velocity slip coefficient are close to the two last theoretical values. In addition, in Ref. [30] the authors tested the influence of the accommodation coefficient on the $\sigma_{2 p}$ coefficient and it was found that this coefficient varies very slightly with accommodation coefficient variations. In our experimental conditions the variation of $\sigma_{2 p}$ coefficient is also weak.

The dependence of $\sigma_{2 p}$ coefficient on the molecular mass of a gas and on the aspect ratio of the channel cross-section was weakly investigated in the literature, see Refs, [31], [32], [33], [16], [34]. The authors of Refs, [33], [16], [34] showed experimentally that $\sigma_{2 p}$ coefficient increases with the molecular mass of a gas. This conclusion was derived by analyzing the gas flow in microchannels of the circular, Refs. [33], [34], and rectangular, Ref. [16], cross-sections. In the present study, the same behavior of the coefficient $\sigma_{2 p}$ increasing with the gas molecular mass is also observed, particularly for the microchannel $A 4$ (see 
Table 9). However, by analyzing globally the behavior of $\sigma_{2 p}$ coefficient, one can notice that the influence of the gas molecular mass is not significant. The values obtained for each microchannel are very similar, almost within their uncertainties, but that could be related to the small variation of $\sigma_{2 p}$ depending on the accommodation of molecules. Taking into account the previous comments we would like to underline that the variation of $\sigma_{2 p}$ coefficient is generally small.

\subsection{Results for non-homogenous walls method}

In Section 4.2, we presented a new generalization of the previously developed methodology [17], which allows to take into account different properties of the vertical and horizontal walls of a microchannel. This difference in the vertical and horizontal walls properties can appear during the fabrication process (channel etching), see Section 2.1, or just due to the wall coating with different materials. The new approach introduces two velocity slip coefficients, $\sigma_{p h}$, and $\sigma_{p w}$, that are related to the properties of the horizontal and vertical walls, respectively.

In this Section, we consider the same experimental data for the channels $A, E$ and $S$ that were used in the previous Sections to extract the velocity slip and tangential momentum accommodation coefficients, to implement the new approach and obtain two velocity slip coefficients and two accommodation coefficients, related to the vertical and horizontal walls. As it was explained in Section 4.2, for the two coefficients $A_{w}$ and $A_{h}$, which are related to the velocity slip coefficients, we have only one equation. Therefore, we have to use all the experimental data from each group of channels together to obtain the $A_{w}$ and $A_{h}$ coefficients, and then, the $\sigma_{p w}$ and $\sigma_{p h}$ coefficients, for that particular group of channels. The obtained results for all groups of channels, $A, E$ and $S$, and two gases, Helium and Argon are given in Tables 14 and 15. Even if the absolute values obtained for group $E$ channels are not sure, we try to detect a systematic relation between same parameters of $A$ and $S$ groups and $E$ groups. In our analysis of the channel of $S$ group we do not included the $S 1$ channel in our analysis, because it was fabricated by different process compared to $S 2-S 4$ channels: namely the channel $S 1$ was made using the half heigh $(h / 2)$ cavities bounding, see Section 2.2 and Fig. 1. We do not include in our analysis the results for Helium, because the determination coefficient for this gas was found very low and therefore the fitting used was not representative for these data.

Before deriving some general tendencies of the accommodation coefficients behavior, we need to note that we do not have any measurements of the vertical wall roughness. However, generally the range of the vertical wall roughness created by DRIE techniques, see Ref. [35], is between $100 \mathrm{~nm}$ and $1 \mu \mathrm{m}$.

We analyzed all groups of channels and only two gases, see Tables 14 and 15, but from these results we can derive some common properties:

1. The tangential momentum accommodation coefficients of the horizontal walls, $\alpha_{h}$, are always greater than those of the vertical walls, $\alpha_{w}$.

2. The tangential momentum accommodation coefficients of the horizontal walls, $\alpha_{h}$, are not very sensitive to the gas nature and these coefficients $\alpha_{h}$ are close to one (near full diffusive reflection of molecules). 
3. The tangential momentum accommodation coefficients of the vertical walls, $\alpha_{w}$, of group $A$ channels are always smaller than those of group $E$ and globally the tangential momentum accommodation coefficients of the vertical walls, $\alpha_{w}$, are not purely diffusive.

4. The observations related to point 1 and the fact that the roughness of the vertical wall is larger than that of the horizontal wall, seem to indicate that in our measurements, the accommodation coefficient increases when the roughness of the surface decreases.

5. For the channels of group A the tangential momentum accommodation coefficient of the vertical wall, $\alpha_{w}$, depends on the nature of the gas. However, in point 2, it was found that the tangential momentum accommodation coefficient of the horizontal walls, $\alpha_{h}$, is not very sensitive to the gas nature. However, the roughness of two walls is very different and probably there is a specific threshold of the roughness value, above which the gas nature starts to influence the accommodation coefficient.

\subsection{Comparison between the results of the first and second methods}

Let us note first that the second method was applied only for two groups of channels, $A$ and $E$. In addition, the quantitative comparison is possible only for the channels of group $A$, because, as explained in Appendix $\mathrm{B}$, the channels of group $E$ are probably affected by a gold layer take off modifying their crosssection. Thus, when considering group A channels, the values of the various accommodation coefficients obtained by the first method for $\mathrm{He}$ and $\mathrm{Ar}$, see Table 9, may be compared to the unique pair of values for $\mathrm{He}$ and $\mathrm{Ar}$, given by the second approach in Table 14. Let us remind the general comment given above in Section 5.1.1, where the various accommodation coefficients of group $A$ channels are found close to each other in the first method and that the aspect ratio has a weak influence. This fact brings a support for that hypothesis used in the second method: an unique pair of coefficients for a group of channels.

Nevertheless, an apparent small contradiction seems to appear about the trend of the accommodation coefficient variation with the roughness variation. Indeed, in point 4 , just here above, we have pointed out that the accommodation coefficient increases when the surface roughness decreases. But, when detailing the roughness in group $\mathrm{A}$, we can observe using the first method, that the $A 4$ channel has highest roughness ( $20 \%$ compared to the other channels in the same group, see Table 1) and highest accommodation coefficient, see Table 9, which seems to contradict the property given here above. In fact, the value given in Table 1 for the roughness, characterizes only the horizontal walls of the channels. Furthermore, we have seen in Section 5.2 that the vertical walls have a higher roughness. Consequently, in the first method, all the unique accommodation coefficients are decreased under this influence. But, in the A4 channel the relative vertical wall influence is the smallest because it has the largest aspect ratio, see Table 1. Therefore, it is not possible to predict and compare the mean roughness really acting on the coefficients of the first method. Thus, the previous comparison may not be considered as significant. 
Finally, the new second method used to analyze the result does not seem to contradict the first method. Conversely, it seems to be a good generalization, able to capture some physical information on the local nature of a surface. The analysis of the results with two different accommodation coefficients of horizontal and vertical walls of a channel, seems to be pertinent if the surface ratio between horizontal and vertical walls remains moderate (weak aspect ratio influence) and if the probable roughness is different between these walls. To use all the advantages, offered by this last approach, we plan to apply it in the future to other type of channels.

\section{Conclusion}

Experimental and theoretical slip regime approaches, developed previously, were applied here to the gas flow through rectangular microchannels of different aspect ratios, materials and roughnesses. Our measurements and modelings allowed us to extract the velocity slip coefficients using the Maxwell type specular-diffuse gas-wall interaction model. We used two theoretical modelings for the flow, taking into account the two-dimensional effect of the rectangular cross-section. The first approach used a single accommodation coefficient for all the walls (horizontal and vertical) of a channel. However, the second approach employed two different accommodation coefficients: $\alpha_{h}$ for the two horizontal walls and another coefficient $\alpha_{w}$ for the two vertical walls. Moreover, this pair of coefficients remains the same for all channels of the same group.

Using the first method (single accommodation coefficient for all walls), it appears that the aspect ratio does not significantly influence the values of the velocity slip and tangential momentum accommodation coefficients and, in a more general way, the physical properties characterizing the flow through the channels of a same surface coating. This result gives credibility to the assumption used in second method: existence of a same pair of accommodation coefficients in each group of channels having the same surface coating.

Considering that the vertical walls have a higher roughness than the horizontal ones, we deduced from the second method that in our roughness range (i.e. for rather low roughness) with the present wall material $(A u)$, the tangential momentum accommodation coefficient decreases when the roughness increases.

In the present range of roughness, using the results obtained with different wall materials, the calculations using the first method show that globally the gold material $(\mathrm{Au})$ is slightly more diffusive than silica $\left(\mathrm{SiO}_{2}\right)$.

We have observed, according the two methods and in our condition of low roughness, that very small changes of the flow properties, notably of the accommodation coefficient occur when the molecular mass of the gas changes.

The previous comments show that the two modeling methods, proposed and employed here, are rather consistent and complementary. The second approach appears to potentially be able to detect some local properties of the gas-surface interaction. Consequently, we plan in the future to develop and to extend the application of this method in various directions: notably in rectangular channels 
having horizontal and vertical walls differing by their wall material or roughness. Furthermore, concerning the roughness influence on the accommodation process, the problem is more general and more complex. It would be interesting to investigate how varies the accommodation beyond the weak roughness studied in the present article; i.e. in a much larger range of roughness, and it would be useful to diversify the wall materials and the flow regimes to conduct these investigations.

\section{Acknowledgments}

We wish to acknowledge the support of MIMENTO platform of technology and especially FEMTO-ST Laboratory (http://www.femto-st.fr) for the microsystems fabrication and the measurement of the microchannels dimensions. This research received funding from the European Community's Seventh Framework Program (ITN FP7/2007-2013) under grant agreement number 215504.

[1] M. Gad-el Hak, Flow physics in mems, Mec. Ind. 2 (2001) 313-341.

[2] A. Agrawal, S. V. Prabhu, Survey on measurement of tangential momentum accommodation coefficient, Journal of Vac. Sci. Technol. A26 (4) (2008) 634-645.

[3] L. Stacy, A determination by the constant deflection method of the value of the coefficient of slip for rough and for smooth surfaces in air, Phys. Rev. 21 (1923) 239-249.

[4] M. Seidl, E. Steinheil, Measurement of momentum accommodation coefficients on surfaces characterized by auger spectroscopy, sims and leed, in: M. Becker, M. Fiebig (Eds.), Rarefied Gas Dynamics, Proceedings of the Ninth International symposium, DFVLR:Porz-Wahn, Germany, 1974, pp. E9.1-E9.12.

[5] L. B. Thomas, R. G. Lord, in: Proceedings of the Eighth Intenational Symposium on Rarefied Gas Dynamics, 1974, pp. 405-412.

[6] D. Blanchard, P. Ligrani, Slip and accommodation coefficients from rarefaction and roughness in rotating microscale disk flows, Physics of Fluids 19 (2007) 063602.

[7] Y. Ji, K. Yuan, J. Chung, Numerical simulation of wall roughness on gaseuous flow and heat transfr in a microchannel, International Journal of Heat and Mass Transfer 49 (2006) 1329-1339.

[8] G. Croce, P. D'Agaro, C. Nonino, Three-dimensional roughness effect on microchannel heat transfer and pressure drop, International Journal Heat and Mass Transfer 50 (2007) 5249-5259. 
[9] H. Sun, M. Faghri, Effect of surface roughness in a microchannel using the direct simulation monte carlo method, Numerical Heat Transfer, part A Appl 43 (1) (2003) 1-8.

[10] O. I. Rovenskaya, Numerical analysis of surface roughness effects on the poiseuille flow caused by a small pressure drop, International Journal Heat and Mass Transfer 110 (2017) 817-826.

[11] Y. Chen, C. Zhang, M. Shi, G. P. Peterson, Slip boundary for fluid flow at rough surface, Applied Physics Letters 100 (2012) 074102.

[12] M. S. Ozhgibesov, T. S. Leu, C. H. Cheng, A. V. Utkin, Studies of argon collisions with smooth and rough tungsten surface, Journal of Molecular Graphics and Modelling 45 (2013) 45-49.

[13] W. Su, Y. Zhang, L. Wu, Rarefaction cloaking: influence of the fractal rough surface in gas slider bearings, Physics of Fluids 29 (2017) 102003.

[14] S. Brull, P. Charrier, L. Mieussens, Nanoscale roughness effect on maxwelllike boundary conditions for the boltzmann equation, Physics of Fluids 28 (2016) 082004.

[15] T. Ewart, P. Perrier, I. A. Graur, J. G. Méolans, Mass flow rate measurements in microchannel, from hydrodynamic to near free molecular regimes, Fluid mechanics 584 (2007) 337-356.

[16] I. A. Graur, P. Perrier, W. Ghozlani, J. G. Méolans, Measurements of tangential momentum accommodation coefficient for various gases in plane microchannel, Physics of Fluids 21 (2009) 102004.

[17] J. G. Méolans, M. H. Nacer, M. Rojas, P. Perrier, I. Graur, Effects of two transversal finite dimensions in long microchannel: Analytical approach in slip regime, Physics of Fluids 24 (2012) 112005.

[18] J. Martya, L. Rousseaua, B. Saadanya, B. Merciera, O. Francaisa, Y. Mitab, T. Bourouina, Advanced etching of silicon based on deep reactive ion etching for silicon high aspect ratio microstructures and threedimensional micro- and nanostructures, Microelectronics 36 (2005) 673677 .

[19] B. Bhushan, Springer Handbook of Nanotechnology, Springer- Verlag, 2007.

[20] J. M. Thevenoud, B. Mercier, T. Bourouina, F. Marty, M. Puech, N. Launay, DRIE technology: from micro to nanoapplications, Tech. rep., Alcatel Micro Machining System (2012). URL http://www.alcatelmicromachining.com/all/dyn/home/

[21] T. Ewart, P. Perrier, I. A. Graur, J. G. Méolans, Mass flow rate measurements in gas micro flows, Experiments in Fluids 41 (3) (2006) 487-498. 
[22] G. A. Bird, Molecular Gas Dynamics and the Direct Simulation of Gas Flows, Oxford Science Publications, Oxford University Press Inc., New York, 1994.

[23] C. Cercignani, Higher order slip according to the linearized Boltzmann equation, Institute of Engineering Research Rep. as-64-19. University of California, Berkeley., Tech. rep. (1964).

[24] S. Cercignani, C. Lorenzani, Variational derivation of second-order slip coefficients on the basis of the boltzmann equation for hard-sphere molecules, Physics of Fluids 22 (2010) 062004.

[25] S. K. Loyalka, N. Petrellis, S. T. Stvorick, Some numerical results for the bgk model: thermal creep and viscous slip problems with arbitrary accommodation of the surface, Physics of Fluids 18 (1975) 1094.

[26] F. Sharipov, Rarefied gas flow through a long tube at arbitrary pressure and temperature drop, J. Vac. Sci. Technol. A 15 (4) (1997) 2434-2436.

[27] C. Cercignani, A. Daneri, Flow of a rarefied gas between two parallel plates, Physics of Fluids 6 (1963) 993-996.

[28] R. G. Deissler, An analysis of second order slip flow and temperature jump boundary conditions for rarefied gas, International Journal Heat and Mass Transfer 45 (1964) 681-694.

[29] N. G. Hadjiconstantinou, Comment on cercignani's second-order slip coefficient, Physics of Fluids 15 (8) (2003) 257-274.

[30] S. Lorenzani, High order slip according to the linearized boltzmann equation with general boundary conditions, Philosophical transactions of Royal Society A 369 (2011) 2228-2236.

[31] C. Cercignani, Mathematical methods in kinetic theory, Premium Press, New York, London, 1990.

[32] Y. Sone, Molecular Gas Dynamics, Birkhäuser, Boston, 2007.

[33] T. Ewart, P. Perrier, I. A. Graur, J. G. Méolans, Tangential momentum accomodation in microtube, Microfluid and Nanofluid 3 (6) (2007) 689-695.

[34] P. Perrier, I. A. Graur, T. Ewart, J. G. Méolans, Mass flow rate measurements in microtubes: From hydrodynamic to near free molecular regime, Physics of Fluids 23 (2011) 042004.

[35] I.-H. Song, P. Y-A, M. Meunier, Smoothing dry-etched microstructure sidewalls using focused ion beam milling for optical applications, Journal of Micromechanics and Microengineering 17 (2007) 1593-1597. 


\begin{tabular}{ccccc}
\hline \hline Microchannel & \multicolumn{2}{c}{$A 1$} & \multicolumn{2}{c}{$A 2$} \\
\hline \hline Quantity & Min & Max & Min & Max \\
\hline Mass flow rate $\dot{M}^{\text {exp }}\left(10^{-12} \mathrm{~kg} / \mathrm{s}\right)$ & 0.4 & 16000 & 0.1 & 34400 \\
\hline Inlet pressure $p_{\text {in }}(\mathrm{Pa})$ & 142.3 & 136760 & 14.4 & 129190 \\
\hline Outlet pressure $p_{\text {out }}(\mathrm{Pa})$ & 28.4 & 88220 & 3.3 & 93300 \\
\hline Average Knudsen number $K n_{m}$ & $1.710^{-3}$ & 6.8 & $1.810^{-3}$ & 70.4 \\
\hline \hline
\end{tabular}

Table 2: Experimental conditions for the microchannels $A 1$ and $A 2$.

\begin{tabular}{ccccc}
\hline \hline Microchannel & \multicolumn{2}{c}{$A 3$} & \multicolumn{2}{c}{$A 4$} \\
\hline \hline Quantity & Min & Max & Min & Max \\
\hline Mass flow rate $\dot{M}^{\text {exp }}\left(10^{-12} \mathrm{~kg} / \mathrm{s}\right)$ & 1.9 & 135000 & 3.4 & 285000 \\
\hline Inlet pressure $p_{\text {in }}(\mathrm{Pa})$ & 61.6 & 125100 & 64.9 & 120250 \\
\hline Outlet pressure $p_{\text {out }}(\mathrm{Pa})$ & 22.1 & 79200 & 25.7 & 79500 \\
\hline Average Knudsen number $K n_{m}$ & $1.710^{-3}$ & 14.7 & $2.110^{-3}$ & 14.6 \\
\hline \hline
\end{tabular}

Table 3: Experimental conditions for the microchannels $A 3$ and $A 4$.

\begin{tabular}{ccccc}
\hline Microchannel & \multicolumn{2}{c}{$E 1$} & \multicolumn{2}{c}{$E 2$} \\
\hline \hline Quantity & Min & Max & Min & Max \\
\hline Mass flow rate $\dot{M}^{\text {exp }}\left(10^{-12} \mathrm{~kg} / \mathrm{s}\right)$ & 0.9 & 21200 & 0.8 & 66100 \\
\hline Inlet pressure $p_{\text {in }}(\mathrm{Pa})$ & 342.1 & 131850 & 139.4 & 129720 \\
\hline Outlet pressure $p_{\text {out }}(\mathrm{Pa})$ & 89.7 & 94900 & 23.1 & 89600 \\
\hline Average Knudsen number $K n_{m}$ & $1.110^{-3}$ & 4.3 & $1.410^{-3}$ & 5.6 \\
\hline
\end{tabular}

Table 4: Experimental conditions for the microchannels $E 1$ and $E 2$.

\begin{tabular}{ccccc}
\hline Microchannel & \multicolumn{2}{c}{$E 3$} & \multicolumn{2}{c}{$E 4$} \\
\hline \hline Quantity & Min & Max & Min & Max \\
\hline Mass flow rate $\dot{M}^{\text {exp }}\left(10^{-12} \mathrm{~kg} / \mathrm{s}\right)$ & 2.1 & 253000 & 2.3 & 574000 \\
\hline Inlet pressure $p_{\text {in }}(\mathrm{Pa})$ & 63.7 & 105850 & 27.2 & 114650 \\
\hline Outlet pressure $p_{\text {out }}(\mathrm{Pa})$ & 22.4 & 74000 & 8.8 & 80000 \\
\hline Average Knudsen number $K n_{m}$ & $6.610^{-4}$ & 11.36 & $1.610^{-3}$ & 27.7 \\
\hline
\end{tabular}

Table 5: Experimental conditions for the microchannels $E 3$ and E4. 


\begin{tabular}{ccccc}
\hline Microchannel & \multicolumn{2}{c}{$S 1$} & \multicolumn{2}{c}{$S 2$} \\
\hline \hline Quantity & Min & Max & Min & Max \\
\hline Mass flow rate $\dot{M}^{\text {exp }}\left(10^{-12} \mathrm{~kg} / \mathrm{s}\right)$ & 0.4 & 10220 & 1.2 & 174600 \\
\hline Inlet pressure $p_{\text {in }}(\mathrm{Pa})$ & 234.8 & 130220 & 105.6 & 125000 \\
\hline Outlet pressure $p_{\text {out }}(\mathrm{Pa})$ & 57.5 & 89850 & 26.5 & 31460 \\
\hline Average Knudsen number $K n_{m}$ & $2.010^{-3}$ & 4.8 & $1.610^{-3}$ & 6.0 \\
\hline
\end{tabular}

Table 6: Experimental conditions for the microchannels $S 1$ and $S 2$.

\begin{tabular}{ccccc}
\hline Microchannel & \multicolumn{2}{c}{$S 3$} & \multicolumn{2}{c}{$S 4$} \\
\hline \hline Quantity & Min & Max & Min & Max \\
\hline Mass flow rate $\dot{M}^{\text {exp }}\left(10^{-12} \mathrm{~kg} / \mathrm{s}\right)$ & 4.3 & 372400 & 3.9 & 952700 \\
\hline Inlet pressure $p_{\text {in }}(\mathrm{Pa})$ & 66.3 & 131000 & 25.9 & 124380 \\
\hline Outlet pressure $p_{\text {out }}(\mathrm{Pa})$ & 22.9 & 108650 & 9.1 & 93300 \\
\hline Average Knudsen number $K n_{m}$ & $1.110^{-4}$ & 9.3 & $1.210^{-4}$ & 24.0 \\
\hline
\end{tabular}

Table 7: Experimental conditions for the microchannels $S 3$ and $S 4$.

\section{Appendix A. Useful expressions}

To provide the complete definition of all functions used in the analytical derivation we give here the expressions for functions $\left(\phi_{n}\right)_{0},\left(\chi_{n}\right)_{0}$ and $\left(\varphi_{n}\right)_{0}$, which can be found also in Appendix of Ref. [17]:

$$
\left(\phi_{n}\right)_{0}=\frac{4(-1)^{n}}{k_{n}^{3}} \cos \left(k_{n} y / h\right) \frac{\cosh \left(k_{n} x / h\right)-\cosh \left(\omega_{n}\right)}{\cosh \left(\omega_{n}\right)},
$$

where $k_{n}=\pi(2 n+1)$ and $\omega_{n}=k_{n} w /(2 h)$.

$$
\left(\chi_{n}\right)_{0}=\frac{2(-1)^{n+1}}{k_{n}^{2} \cosh \left(\omega_{n}\right)} \tanh \left(\omega_{n}\right) \cos \left(k_{n} y / h\right) \cosh \left(k_{n} x / h\right)
$$

$$
\begin{aligned}
& \left(\varphi_{n}\right)_{0}=\frac{2(-1)^{n+1}}{k_{n} \cosh \left(\omega_{n}\right)}\left(\cosh \left(\omega_{n}\right)-\cosh \left(k_{n} x / h\right)\right) \times \\
& \left(\frac{4}{k_{n}} \cos \left(k_{n} y / h\right)+\frac{2 y}{h} \sin \left(k_{n} y / h\right)\right)- \\
& \cos \left(k_{n} y / h\right)\left(\frac{2 x}{h} \sinh \left(k_{n} x / h\right)-\frac{w}{h} \sinh \left(\omega_{n}\right)\right)- \\
& \frac{w}{h} \tanh \left(\omega_{n}\right) \cos \left(k_{n} y / h\right)\left(\cosh \left(k_{n} x / h\right)-\cosh \left(\omega_{n}\right)\right) .
\end{aligned}
$$




\begin{tabular}{|c|c|c|c|c|c|c|c|}
\hline Channel & Gas & $B_{0}^{e x p}$ & $B_{1}^{\text {exp }}$ & $B_{2}^{e x p}$ & $s_{r}$ & $\overline{r^{2}}$ & $E_{s}(\%)$ \\
\hline \multirow[t]{6}{*}{$A 1$} & \multirow[t]{2}{*}{$\mathrm{He}$} & $1.023 \pm 0.008$ & $10.625 \pm 0.165$ & - & 0.021 & 0.996 & 1.4 \\
\hline & & $1.019 \pm 0.007$ & $10.666 \pm 0.142$ & $1.635 \pm 0.536$ & 0.020 & 0.999 & 0.9 \\
\hline & \multirow[t]{2}{*}{$N_{2}$} & $1.009 \pm 0.005$ & $12.104 \pm 0.111$ & - & 0.016 & 0.998 & 1.1 \\
\hline & & $1.008 \pm 0.006$ & $12.181 \pm 0.171$ & $-1.555 \pm 0.850$ & 0.020 & 0.999 & 1.1 \\
\hline & \multirow[t]{2}{*}{$A r$} & $1.012 \pm 0.009$ & $12.621 \pm 0.190$ & - & 0.028 & 0.995 & 1.9 \\
\hline & & $1.027 \pm 0.014$ & $11.731 \pm 0.390$ & $3.115 \pm 1.863$ & 0.044 & 0.997 & 2.3 \\
\hline \multirow[t]{6}{*}{$A 2$} & \multirow[t]{2}{*}{$\mathrm{He}$} & $1.001 \pm 0.003$ & $8.785 \pm 0.056$ & - & 0.008 & 0.999 & 0.6 \\
\hline & & $1.000 \pm 0.008$ & $8.681 \pm 0.183$ & $2.324 \pm 0.729$ & 0.024 & 0.999 & 1.3 \\
\hline & \multirow[t]{2}{*}{$N_{2}$} & $0.996 \pm 0.004$ & $9.168 \pm 0.098$ & - & 0.015 & 0.997 & 1.1 \\
\hline & & $0.999 \pm 0.008$ & $8.701 \pm 0.195$ & $7.575 \pm 0.812$ & 0.027 & 0.999 & 1.5 \\
\hline & \multirow[t]{2}{*}{$A r$} & $1.017 \pm 0.005$ & $9.580 \pm 0.112$ & - & 0.016 & 0.997 & 1.1 \\
\hline & & $1.014 \pm 0.006$ & $9.448 \pm 0.157$ & $6.699 \pm 0.645$ & 0.021 & 0.999 & 1.1 \\
\hline \multirow[t]{6}{*}{$\overline{A 3}$} & \multirow[t]{2}{*}{$\mathrm{He}$} & $1.011 \pm 0.002$ & $8.699 \pm 0.046$ & - & 0.006 & 0.999 & 0.5 \\
\hline & & $1.013 \pm 0.003$ & $8.426 \pm 0.062$ & $3.981 \pm 0.237$ & 0.009 & 0.999 & 0.5 \\
\hline & \multirow[t]{2}{*}{$N_{2}$} & $1.003 \pm 0.002$ & $9.413 \pm 0.042$ & - & 0.007 & 0.999 & 0.5 \\
\hline & & $1.006 \pm 0.002$ & $9.047 \pm 0.038$ & $4.944 \pm 0.144$ & 0.006 & 0.999 & 0.3 \\
\hline & \multirow[t]{2}{*}{$A r$} & $1.013 \pm 0.003$ & $10.078 \pm 0.068$ & - & 0.011 & 0.999 & 0.8 \\
\hline & & $1.017 \pm 0.002$ & $9.593 \pm 0.060$ & $5.767 \pm 0.256$ & 0.008 & 0.999 & 0.5 \\
\hline \multirow[t]{6}{*}{$A 4$} & \multirow[t]{2}{*}{$\mathrm{He}$} & $0.988 \pm 0.003$ & $7.993 \pm 0.073$ & - & 0.008 & 0.998 & 0.6 \\
\hline & & $0.984 \pm 0.005$ & $7.974 \pm 0.106$ & $4.072 \pm 0.442$ & 0.014 & 0.999 & 0.8 \\
\hline & \multirow[t]{2}{*}{$N_{2}$} & $0.989 \pm 0.003$ & $9.110 \pm 0.080$ & - & 0.010 & 0.998 & 0.8 \\
\hline & & $0.993 \pm 0.005$ & $8.660 \pm 0.121$ & $6.555 \pm 0.464$ & 0.018 & 0.999 & 1.0 \\
\hline & \multirow[t]{2}{*}{$A r$} & $1.007 \pm 0.003$ & $9.326 \pm 0.074$ & - & 0.012 & 0.998 & 0.9 \\
\hline & & $1.008 \pm 0.007$ & $8.784 \pm 0.177$ & $9.661 \pm 0.670$ & 0.025 & 0.999 & 1.4 \\
\hline
\end{tabular}

Table 8: Fitting coefficients of the dimensionless mass flow rate $S^{\exp }$ obtained with the first (first line) and second order (second line) approximations (44) in the mean Knudsen number ranges $[0,0.1]$ and $[0,0.3]$, respectively, for microchannels of the group $A$. The last three columns in this Table are: $s_{r}$ is the squared residual sum, $r^{2}$ is the determination coefficient and $E_{s}(\%)$ is the standard error, see Section 4.1 for more details. We remind the dimensions of the microchannel $A 1(h=27.8 \mu \mathrm{m}, w=52.2 \mu \mathrm{m}) ; A 2(h=27.6 \mu \mathrm{m}, w=107.6 \mu \mathrm{m}) ; A 3$ $(h=27.9 \mu \mathrm{m}, w=504.0 \mu \mathrm{m}) ; A 4(h=25.8 \mu \mathrm{m}, w=1005.5 \mu \mathrm{m})$, see Table 1 . 


\begin{tabular}{|c|c|c|c|c|c|}
\hline Channel & Gas & molecular mass $(\mathrm{g} / \mathrm{mol})$ & $\sigma_{p}$ & $\alpha$ & $\sigma_{2 p}$ \\
\hline \multirow[t]{6}{*}{$A 1$} & $\mathrm{He}$ & 4.00 & $1.263 \pm 0.020$ & $0.885 \pm 0.008$ & - \\
\hline & & & $1.268 \pm 0.017$ & $0.883 \pm 0.007$ & $0.053 \pm 0.018$ \\
\hline & $N_{2}$ & 28.02 & $1.338 \pm 0.012$ & $0.855 \pm 0.005$ & - \\
\hline & & & $1.346 \pm 0.019$ & $0.851 \pm 0.007$ & $-0.043 \pm 0.023$ \\
\hline & $A r$ & 39.95 & $1.305 \pm 0.020$ & $0.867 \pm 0.008$ & - \\
\hline & & & $1.213 \pm 0.040$ & $0.906 \pm 0.018$ & $0.075 \pm 0.045$ \\
\hline \multirow[t]{6}{*}{$A 2$} & $\mathrm{He}$ & 4.00 & $\bar{~} 1.109 \pm 0.007$ & $0.953 \pm 0.003$ & - \\
\hline & & & $1.096 \pm 0.023$ & $0.960 \pm 0.011$ & $0.095 \pm 0.030$ \\
\hline & $N_{2}$ & 28.02 & $1.076 \pm 0.011$ & $0.969 \pm 0.006$ & - \\
\hline & & & $1.021 \pm 0.023$ & $0.997 \pm 0.012$ & $0.265 \pm 0.028$ \\
\hline & $A r$ & 39.95 & $1.052 \pm 0.012$ & $0.981 \pm 0.006$ & - \\
\hline & & & $1.038 \pm 0.017$ & $0.989 \pm 0.009$ & $0.206 \pm 0.020$ \\
\hline \multirow[t]{6}{*}{$\overline{A B 3}$} & $\mathrm{He}$ & 4.00 & 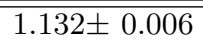 & $0.943 \pm 0.003$ & - \\
\hline & & & $1.096 \pm 0.008$ & $0.960 \pm 0.004$ & $0.192 \pm 0.011$ \\
\hline & $N_{2}$ & 28.02 & $1.138 \pm 0.005$ & $0.940 \pm 0.002$ & - \\
\hline & & & $1.094 \pm 0.005$ & $0.961 \pm 0.002$ & $0.203 \pm 0.006$ \\
\hline & $A r$ & 39.95 & $1.141 \pm 0.008$ & $0.938 \pm 0.004$ & - \\
\hline & & & $1.086 \pm 0.007$ & $0.965 \pm 0.003$ & $0.208 \pm 0.009$ \\
\hline \multirow[t]{6}{*}{$A 4$} & $\mathrm{He}$ & 4.00 & 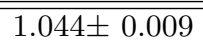 & 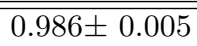 & - \\
\hline & & & $1.041 \pm 0.014$ & $0.987 \pm 0.007$ & $0.198 \pm 0.022$ \\
\hline & $N_{2}$ & 28.02 & $1.106 \pm 0.010$ & $0.955 \pm 0.005$ & - \\
\hline & & & $1.051 \pm 0.015$ & $0.982 \pm 0.007$ & $0.272 \pm 0.019$ \\
\hline & $A r$ & 39.95 & $1.060 \pm 0.008$ & $0.978 \pm 0.004$ & - \\
\hline & & & $0.998 \pm 0.020$ & $1.009 \pm 0.011$ & $0.351 \pm 0.024$ \\
\hline
\end{tabular}

Table 9: Slip and tangential momentum accommodation coefficients found experimentally from the first (first line) and second order (second line) approximations of the dimensionless mass flow rate $S^{\exp }(44)$, respectively, for microchannels of the group $A$. We remind the dimensions of the microchannel $A 1(h=27.8 \mu \mathrm{m}, w=52.2 \mu \mathrm{m}) ; A 2(h=27.6 \mu \mathrm{m}, w=$ $107.6 \mu \mathrm{m}) ; A 3(h=27.9 \mu \mathrm{m}, w=504.0 \mu \mathrm{m}) ; A 4(h=25.8 \mu \mathrm{m}, w=1005.5 \mu \mathrm{m})$, see Table 1 . 


\begin{tabular}{|c|c|c|c|c|c|c|c|}
\hline Channel & Gas & $B_{0}^{\text {exp }}$ & $B_{1}^{e x p}$ & $B_{2}^{\exp }$ & $s_{r}$ & $r^{2}$ & $E_{s}(\%)$ \\
\hline \multirow[t]{6}{*}{$E 1$} & \multirow[t]{2}{*}{$\mathrm{He}$} & $1.042 \pm 0.001$ & $8.133 \pm 0.032$ & - & 0.005 & 0.999 & 0.4 \\
\hline & & $1.042 \pm 0.003$ & $7.978 \pm 0.061$ & $3.081 \pm 0.244$ & 0.009 & 0.999 & 0.5 \\
\hline & \multirow[t]{2}{*}{$N_{2}$} & $1.037 \pm 0.001$ & $9.333 \pm 0.033$ & - & 0.005 & 0.999 & 0.4 \\
\hline & & $1.042 \pm 0.003$ & $8.926 \pm 0.083$ & $4.359 \pm 0.329$ & 0.013 & 0.999 & 0.7 \\
\hline & \multirow[t]{2}{*}{$A r$} & $1.048 \pm 0.001$ & $10.146 \pm 0.035$ & - & 0.005 & 0.999 & 0.4 \\
\hline & & $1.054 \pm 0.002$ & $9.562 \pm 0.053$ & $6.682 \pm 0.233$ & 0.007 & 0.999 & 0.4 \\
\hline \multirow[t]{6}{*}{$E 2$} & \multirow[t]{2}{*}{$\mathrm{He}$} & $1.030 \pm 0.001$ & $7.227 \pm 0.025$ & - & 0.004 & 0.999 & 0.3 \\
\hline & & $1.032 \pm 0.001$ & $6.972 \pm 0.024$ & $3.177 \pm 0.098$ & 0.003 & 0.999 & 0.2 \\
\hline & \multirow[t]{2}{*}{$N_{2}$} & $1.027 \pm 0.001$ & $8.103 \pm 0.023$ & - & 0.004 & 0.999 & 0.3 \\
\hline & & $1.026 \pm 0.003$ & $7.976 \pm 0.066$ & $2.880 \pm 0.269$ & 0.010 & 0.999 & 0.6 \\
\hline & \multirow[t]{2}{*}{$A r$} & $1.039 \pm 0.001$ & $8.704 \pm 0.035$ & - & 0.006 & 0.999 & 0.4 \\
\hline & & $1.039 \pm 0.003$ & $8.599 \pm 0.073$ & $2.536 \pm 0.302$ & 0.011 & 0.999 & 0.7 \\
\hline \multirow[t]{6}{*}{$E 3$} & \multirow[t]{2}{*}{$\mathrm{He}$} & $1.036 \pm 0.001$ & $6.728 \pm 0.022$ & - & 0.003 & 0.999 & 0.3 \\
\hline & & $1.039 \pm 0.001$ & $6.424 \pm 0.022$ & $3.810 \pm 0.090$ & 0.003 & 0.999 & 0.2 \\
\hline & \multirow[t]{2}{*}{$N_{2}$} & $1.033 \pm 0.001$ & $7.572 \pm 0.025$ & - & 0.004 & 0.999 & 0.3 \\
\hline & & $1.034 \pm 0.001$ & $7.354 \pm 0.032$ & $3.508 \pm 0.130$ & 0.005 & 0.999 & 0.3 \\
\hline & \multirow[t]{2}{*}{$A r$} & $1.045 \pm 0.001$ & $8.220 \pm 0.034$ & - & 0.005 & 0.999 & 0.4 \\
\hline & & $1.046 \pm 0.001$ & $7.972 \pm 0.033$ & $3.773 \pm 0.134$ & 0.005 & 0.999 & 0.3 \\
\hline \multirow[t]{6}{*}{$E 4$} & \multirow[t]{2}{*}{$\mathrm{He}$} & $1.054 \pm 0.002$ & $6.839 \pm 0.044$ & - & 0.007 & 0.999 & 0.5 \\
\hline & & $1.056 \pm 0.003$ & $6.612 \pm 0.074$ & $3.223 \pm 0.290$ & 0.011 & 0.999 & 0.7 \\
\hline & \multirow[t]{2}{*}{$N_{2}$} & $1.055 \pm 0.003$ & $7.614 \pm 0.078$ & - & 0.011 & 0.997 & 0.9 \\
\hline & & $1.055 \pm 0.005$ & $7.344 \pm 0.135$ & $4.930 \pm 0.546$ & 0.020 & 0.999 & 1.2 \\
\hline & \multirow[t]{2}{*}{$A r$} & $1.062 \pm 0.003$ & $8.414 \pm 0.071$ & - & 0.010 & 0.998 & 0.8 \\
\hline & & $1.066 \pm 0.006$ & $7.980 \pm 0.160$ & $5.238 \pm 0.641$ & 0.024 & 0.999 & 1.4 \\
\hline
\end{tabular}

Table 10: Fitting coefficients of the dimensionless mass flow rate $S^{\exp }$ obtained with the first (first line) and second order (second line) approximations in the mean Knudsen number ranges $[0.0,0.1]$ and $[0.0,0.3]$, respectively, for microchannels of the group $E$. The last three columns in this Table are: $s_{r}$ is the squared residual sum, $r^{2}$ is the determination coefficient and $E_{s}(\%)$ is the standard error, see Section 4.1 for more details. We remind the dimensions of the microchannel $E 1(h=33.5 \mu \mathrm{m}, w=55.5 \mu \mathrm{m}) ; E 2(h=35.2 \mu \mathrm{m}, w=103.8 \mu \mathrm{m}) ; E 3$ $(h=34.9 \mu \mathrm{m}, w=505.0 \mu \mathrm{m}) ; E 4(h=34.2 \mu \mathrm{m}, \mathrm{w}=1001.3 \mu \mathrm{m})$, see Table 1. 


\begin{tabular}{|c|c|c|c|c|c|}
\hline Channel & Gas & molecular mass $(\mathrm{g} / \mathrm{mol})$ & $\sigma_{p}$ & $\alpha$ & $\sigma_{2 p}$ \\
\hline \multirow[t]{6}{*}{$E 1$} & $\mathrm{He}$ & 4.00 & $0.947 \pm 0.004$ & $1.038 \pm 0.002$ & - \\
\hline & & & $0.928 \pm 0.007$ & $1.048 \pm 0.004$ & $0.094 \pm 0.007$ \\
\hline & $N_{2}$ & 28.02 & $1.010 \pm 0.004$ & $1.003 \pm 0.002$ & - \\
\hline & & & $0.966 \pm 0.009$ & $1.027 \pm 0.005$ & $0.115 \pm 0.009$ \\
\hline & $A r$ & 39.95 & $1.027 \pm 0.004$ & $0.994 \pm 0.002$ & - \\
\hline & & & $0.968 \pm 0.005$ & $1.026 \pm 0.003$ & $0.153 \pm 0.005$ \\
\hline \multirow[t]{6}{*}{$\overline{E E}$} & $\mathrm{He}$ & 4.00 & $0.899 \pm 0.003$ & $1.065 \pm 0.002$ & - \\
\hline & & & $0.867 \pm 0.003$ & $1.084 \pm 0.002$ & $0.123 \pm 0.004$ \\
\hline & $N_{2}$ & 28.02 & $0.937 \pm 0.003$ & $1.043 \pm 0.002$ & - \\
\hline & & & $0.922 \pm 0.008$ & $1.051 \pm 0.004$ & $0.095 \pm 0.009$ \\
\hline & $A r$ & 39.95 & $0.942 \pm 0.004$ & $1.040 \pm 0.002$ & - \\
\hline & & & $0.931 \pm 0.008$ & $1.047 \pm 0.005$ & $0.074 \pm 0.009$ \\
\hline \multirow[t]{6}{*}{$\overline{E E 3}$} & $\mathrm{He}$ & 4.00 & $0.874 \pm 0.003$ & $1.080 \pm 0.002$ & - \\
\hline & & & $0.834 \pm 0.003$ & $1.104 \pm 0.002$ & $0.181 \pm 0.004$ \\
\hline & $N_{2}$ & 28.02 & $0.914 \pm 0.003$ & $1.056 \pm 0.002$ & - \\
\hline & & & $0.888 \pm 0.004$ & $1.072 \pm 0.002$ & $0.142 \pm 0.005$ \\
\hline & $A r$ & 39.95 & $0.929 \pm 0.004$ & $1.048 \pm 0.002$ & - \\
\hline & & & $0.901 \pm 0.004$ & $1.064 \pm 0.002$ & $0.133 \pm 0.005$ \\
\hline \multirow[t]{6}{*}{$E 4$} & $\mathrm{He}$ & 4.00 & $0.892 \pm 0.006$ & $1.069 \pm 0.003$ & - \\
\hline & & & $0.863 \pm 0.010$ & $1.087 \pm 0.006$ & $0.155 \pm 0.014$ \\
\hline & $N_{2}$ & 28.02 & $0.923 \pm 0.009$ & $1.051 \pm 0.005$ & - \\
\hline & & & $0.891 \pm 0.016$ & $1.070 \pm 0.010$ & $0.203 \pm 0.022$ \\
\hline & $A r$ & 39.95 & $0.955 \pm 0.008$ & $1.033 \pm 0.004$ & - \\
\hline & & & $0.906 \pm 0.018$ & $1.061 \pm 0.011$ & $0.189 \pm 0.023$ \\
\hline
\end{tabular}

Table 11: Slip and tangential momentum accommodation coefficients found experimentally from the first (first line) and second order (second line) approximations of the dimensionless mass flow rate $S^{e x p}$, for microchannels of the group $E$. We remind the dimensions of the microchannel $E 1(h=33.5 \mu \mathrm{m}, w=55.5 \mu \mathrm{m}) ; E 2(h=35.2 \mu \mathrm{m}, w=103.8 \mu \mathrm{m}) ; E 3(h=$ $34.9 \mu \mathrm{m}, w=505.0 \mu \mathrm{m}) ; E 4(h=34.2 \mu \mathrm{m}, \mathrm{w}=1001.3 \mu \mathrm{m})$, see Table 1 . 


\begin{tabular}{|c|c|c|c|c|c|c|c|}
\hline Channel & Gas & $B_{0}^{\text {exp }}$ & $B_{1}^{e x p}$ & $B_{2}^{\exp }$ & $s_{r}$ & $r^{2}$ & $E_{s}(\%)$ \\
\hline \multirow[t]{6}{*}{$S 1$} & $\mathrm{He}$ & $0.989 \pm 0.003$ & $9.380 \pm 0.072$ & - & 0.010 & 0.999 & 0.7 \\
\hline & & $0.988 \pm 0.004$ & $9.222 \pm 0.085$ & $3.497 \pm 0.315$ & 0.013 & 0.999 & 0.7 \\
\hline & $N_{2}$ & $0.993 \pm 0.003$ & $10.203 \pm 0.062$ & - & 0.009 & 0.999 & 0.7 \\
\hline & & $0.984 \pm 0.009$ & $10.421 \pm 0.220$ & $3.158 \pm 0.866$ & 0.032 & 0.999 & 1.8 \\
\hline & $A r$ & $1.000 \pm 0.005$ & $10.983 \pm 0.112$ & - & 0.017 & 0.997 & 1.2 \\
\hline & & $0.991 \pm 0.011$ & $11.098 \pm 0.286$ & $4.876 \pm 1.138$ & 0.042 & 0.998 & 2.2 \\
\hline \multirow[t]{6}{*}{$S 2$} & $\mathrm{He}$ & $0.997 \pm 0.007$ & $9.582 \pm 0.135$ & - & 0.017 & 0.996 & 1.1 \\
\hline & & $0.997 \pm 0.007$ & $9.325 \pm 0.147$ & $4.825 \pm 0.561$ & 0.018 & 0.999 & 0.9 \\
\hline & $N_{2}$ & $0.992 \pm 0.004$ & $10.941 \pm 0.082$ & - & 0.013 & 0.998 & 0.9 \\
\hline & & $0.989 \pm 0.004$ & $10.868 \pm 0.104$ & $4.101 \pm 0.410$ & 0.016 & 0.999 & 0.9 \\
\hline & $A r$ & $1.007 \pm 0.003$ & $11.493 \pm 0.082$ & - & 0.013 & 0.998 & 0.9 \\
\hline & & $1.008 \pm 0.005$ & $11.313 \pm 0.134$ & $3.289 \pm 0.537$ & 0.021 & 0.999 & 1.1 \\
\hline \multirow[t]{6}{*}{$S 3$} & $\mathrm{He}$ & $0.998 \pm 0.002$ & $9.468 \pm 0.033$ & - & 0.006 & 0.999 & 0.4 \\
\hline & & $1.002 \pm 0.002$ & $9.086 \pm 0.042$ & $4.918 \pm 0.173$ & 0.006 & 0.999 & 0.4 \\
\hline & $N_{2}$ & $0.994 \pm 0.003$ & $10.085 \pm 0.083$ & - & 0.012 & 0.998 & 0.9 \\
\hline & & $0.993 \pm 0.003$ & $9.914 \pm 0.082$ & $5.369 \pm 0.357$ & 0.012 & 0.999 & 0.7 \\
\hline & $A r$ & $1.006 \pm 0.003$ & $11.071 \pm 0.080$ & - & 0.011 & 0.998 & 0.8 \\
\hline & & $1.009 \pm 0.003$ & $10.722 \pm 0.074$ & $4.560 \pm 0.315$ & 0.011 & 0.999 & 0.6 \\
\hline \multirow[t]{6}{*}{$S 4$} & $\mathrm{He}$ & $0.995 \pm 0.002$ & $9.515 \pm 0.039$ & - & 0.006 & 0.999 & 0.5 \\
\hline & & $0.996 \pm 0.003$ & $9.266 \pm 0.080$ & $4.015 \pm 0.345$ & 0.011 & 0.999 & 0.6 \\
\hline & $N_{2}$ & $0.991 \pm 0.002$ & $10.096 \pm 0.042$ & - & 0.006 & 0.999 & 0.5 \\
\hline & & $0.993 \pm 0.002$ & $9.822 \pm 0.049$ & $4.284 \pm 0.225$ & 0.007 & 0.999 & 0.4 \\
\hline & $A r$ & $1.002 \pm 0.003$ & $10.654 \pm 0.071$ & - & 0.011 & 0.999 & 0.8 \\
\hline & & $1.003 \pm 0.003$ & $10.386 \pm 0.076$ & $4.699 \pm 0.330$ & 0.011 & 0.999 & 0.7 \\
\hline
\end{tabular}

Table 12: Fitting coefficients of the dimensionless mass flow rate $S^{\exp }$ obtained with the first (first line) and second order (second line) approximations in the mean Knudsen number ranges $[0.0,0.1]$ and $[0.0,0.3]$, respectively, for microchannels of the group $S$. The last three columns in this Table are: $s_{r}$ is the squared residual sum, $r^{2}$ is the determination coefficient and $E_{s}(\%)$ is the standard error, see Section 4.1 for more details. We remind the dimensions of the microchannel $S 1(h=24.3 \mu \mathrm{m}, w=50.1 \mu \mathrm{m}) ; S 2(h=42.3 \mu \mathrm{m}, w=100.0 \mu \mathrm{m}) ; S 3$ $(h=42.0 \mu \mathrm{m}, w=500.0 \mu \mathrm{m}) ; S 4(h=41.5 \mu \mathrm{m}, \mathrm{w}=1000.0 \mu \mathrm{m})$, see Table 1 . 


\begin{tabular}{|c|c|c|c|c|c|}
\hline Channel & Gas & $\begin{array}{l}\text { molecular mass }(\mathrm{g} / \mathrm{mol}) \\
\end{array}$ & $\sigma_{p}$ & $\alpha$ & $\sigma_{2 p}$ \\
\hline \multirow[t]{6}{*}{$S 1$} & $\overline{\mathrm{H}} \mathrm{He}$ & ( 4.00 & 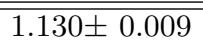 & $0.944 \pm 0.004$ & - \\
\hline & & & $1.111 \pm 0.010$ & $0.953 \pm 0.005$ & $0.120 \pm 0.011$ \\
\hline & $N_{2}$ & 28.02 & $1.142 \pm 0.007$ & $0.938 \pm 0.003$ & - \\
\hline & & & $1.167 \pm 0.025$ & $0.927 \pm 0.011$ & $0.093 \pm 0.025$ \\
\hline & $A r$ & 39.95 & $1.151 \pm 0.012$ & $0.934 \pm 0.005$ & - \\
\hline & & & $1.163 \pm 0.030$ & $0.928 \pm 0.014$ & $0.125 \pm 0.029$ \\
\hline \multirow[t]{6}{*}{$S 2$} & $\mathrm{He}$ & 4.00 & $1.168 \pm 0.016$ & $0.926 \pm 0.007$ & - \\
\hline & & & $1.137 \pm 0.018$ & $0.940 \pm 0.008$ & $0.157 \pm 0.018$ \\
\hline & $N_{2}$ & 28.02 & $1.240 \pm 0.009$ & $0.894 \pm 0.004$ & - \\
\hline & & & $1.232 \pm 0.012$ & $0.898 \pm 0.005$ & $0.115 \pm 0.012$ \\
\hline & $A r$ & 39.95 & $1.219 \pm 0.009$ & $0.903 \pm 0.004$ & - \\
\hline & & & $1.200 \pm 0.014$ & $0.912 \pm 0.006$ & $0.081 \pm 0.013$ \\
\hline \multirow[t]{6}{*}{$S 3$} & $\mathrm{He}$ & 4.00 & $1.227 \pm 0.004$ & $0.900 \pm 0.002$ & - \\
\hline & & & $1.178 \pm 0.005$ & $0.922 \pm 0.002$ & $0.232 \pm 0.008$ \\
\hline & $N_{2}$ & 28.02 & $1.215 \pm 0.010$ & $0.905 \pm 0.004$ & - \\
\hline & & & $1.195 \pm 0.010$ & $0.914 \pm 0.004$ & $0.218 \pm 0.014$ \\
\hline & $A r$ & 39.95 & $1.248 \pm 0.009$ & $0.891 \pm 0.004$ & - \\
\hline & & & $1.209 \pm 0.008$ & $0.908 \pm 0.004$ & $0.161 \pm 0.011$ \\
\hline \multirow[t]{6}{*}{$S 4$} & $\mathrm{He}$ & 4.00 & $1.240 \pm 0.005$ & $0.894 \pm 0.002$ & - \\
\hline & & & $1.208 \pm 0.010$ & $0.908 \pm 0.005$ & $0.196 \pm 0.017$ \\
\hline & $N_{2}$ & 28.02 & $1.223 \pm 0.005$ & $0.902 \pm 0.002$ & - \\
\hline & & & $1.190 \pm 0.006$ & $0.916 \pm 0.003$ & $0.180 \pm 0.009$ \\
\hline & $A r$ & 39.95 & $1.208 \pm 0.008$ & $0.908 \pm 0.004$ & - \\
\hline & & & $1.178 \pm 0.009$ & $0.922 \pm 0.004$ & $0.172 \pm 0.012$ \\
\hline
\end{tabular}

Table 13: Slip and tangential momentum accommodation coefficients found experimentally from the first (first line) and second order (second line) approximations of the dimensionless mass flow rate $S^{\exp }$, respectively, for microchannels of the group $S$. We remind the dimensions of the microchannel $S 1(h=24.3 \mu \mathrm{m}, w=50.1 \mu \mathrm{m}) ; S 2(h=42.3 \mu \mathrm{m}, w=100.0 \mu \mathrm{m}) ; S 3$ $(h=42.0 \mu \mathrm{m}, w=500.0 \mu \mathrm{m}) ; S 4(h=41.5 \mu \mathrm{m}, \mathrm{w}=1000.0 \mu \mathrm{m})$, see Table 1 .

\begin{tabular}{cccccc}
\hline \hline Channels & Gas & $A_{0}$ & $A_{h}$ & $A_{w}$ & $r^{2}$ \\
\hline \hline \multirow{2}{*}{$A 1+A 2+A 3+A 4$} & $H e$ & 1.029 & 1.411 & 1.945 & 0.999 \\
\cline { 2 - 6 } & $A r$ & 1.011 & 1.564 & 2.858 & 0.997 \\
\hline \hline \multirow{2}{*}{$E 1+E 2+E 3+E 4$} & $H e$ & 1.040 & 1.118 & 1.373 & 0.999 \\
\cline { 2 - 6 } & $A r$ & 1.048 & 1.370 & 1.767 & 0.999 \\
\hline \hline$S 2+S 3+S 4$ & $A r$ & 1.032 & 1.654 & 3.645 & 0.930 \\
\hline \hline
\end{tabular}

Table 14: Fitting coefficients of the dimensionless mass flow rate $S^{e x p}$, Eq. (52), obtained with the first order approximations in the mean Knudsen number ranges [0.0, 0.1]. The non-homogenous wall method is used. $r^{2}$ is the determination coefficient. The errors on the coefficients are not provided because they are smaller then $10^{-4}$. 


\begin{tabular}{cccccc}
\hline \hline Channels & Gas & $\sigma_{p h}$ & $\sigma_{p w}$ & $\alpha_{h}$ & $\alpha_{w}$ \\
\hline \hline \multirow{2}{*}{$A 1+A 2+A 3+A 4$} & $H e$ & 1.110 & 1.529 & 0.953 & 0.786 \\
\cline { 2 - 6 } & $A r$ & 1.069 & 1.955 & 0.973 & 0.666 \\
\hline \hline \multirow{2}{*}{$E 1+E 2+E 3+E 4$} & $H e$ & 0.879 & 1.080 & 1.077 & 0.968 \\
\cline { 2 - 6 } & $A r$ & 0.937 & 1.209 & 1.043 & 0.908 \\
\hline \hline$S 2+S 3+S 4$ & $A r$ & 1.131 & 2.493 & 0.943 & 0.557 \\
\hline \hline
\end{tabular}

Table 15: Slip and tangential momentum accommodation coefficients calculated from the measured mass flow rate by using the second approach with two accommodation coefficients, $\alpha_{h}$ and $\alpha_{w}$, for the horizontal and vertical walls, respectively. The results for two groups of microchannels de types $\mathrm{A}, \mathrm{E}$ and $\mathrm{S}$ are presented for two gases $\mathrm{He}$ and $\mathrm{Ar}$, of molecular masses 4.00 and 39.95 , respectively.

\section{Appendix B. Microchannels of group $E$}

From Table 10, one can see that the values of the fitting coefficients, $B_{0}^{e x p}$, obtained with the first and second order polynomial form, are different from the theoretical value $B_{0}^{T}=1$, see Eq. (43). This surprising discrepancy, greater than experimental uncertainty, may be caused by an error in the measurement of microchannels dimensions ( $h$ and/or $w$ ) or by an error on the mass flow rate measurements. The possible reasons for these surprising results are discussed below. Several assumptions are made and analyzed in order to find the causes of such results.

1. Due to the higher value of the coefficients $B_{0}^{\exp }$, compared to 1 , see Table 10, the first explanation on the obtained results is a possible error in the measurements of the microchannels dimensions. By bringing back the values of the coefficients $B_{0}^{e x p}$ to 1 , either by increasing the value of the microchannels height $h$ (or width $w$ ), or both at the same time, the values of coefficient $\sigma_{p}$ decrease and then the values of the coefficient $\alpha$ increase further. Thus, this hypothesis does not seem to solve the problem. In addition, the same method of cross-section measurements is used for the other microchannels of groups $A$ and $S$, where reasonable results were obtained. Therefore, we eliminate the hypothesis of an error in the microchannels dimensions.

2. The second hypothesis is a possibility of an error in the experimental mass flow rate $\dot{M}^{e x p}$, extracted from pressure measurements by using Eq. (4). In this case, there are two possible sources for such kind of errors: first, the measurement errors of the parameters used to calculate mass flow rate (essentially tank volumes), second, a leak or an outgassing problem caused by mishandling.

The possibility of an error in the measurements of the tanks volume was checked and cancelled. Very simple and accurate water weighting technique was used to measure the tanks volume. In addition, the measurements were repeated several times each and the results of these measurements were different within the experimental uncertainty of the volume 
(less than 2.0\%).

The second possible source of the problems is the outgassing or leak. The outgassing and the leak are checked before each experiment, then these two factors cannot be the sources of problems.

3. In the same time, multiple reasons push us to think that the gold layer takeoff is the cause of $B_{0}^{e x p}$ coefficient and the $\alpha$ values to be different from their theoretical values:

- First, after eliminating all the previous hypotheses, it is the only one that seems to be reasonable and tangible.

- Second, during the preliminary tests of microchannels we have encountered similar problem of gold layer takeoff with other microchannels, where the takeoff blocked completely the microchannels.

For the microchannels $E$ we think that the takeoff happened inside the microchannels, that is why the verification which was conducted with the microscope did not reveal anything. Further verification would involve the use of very expensive cutting technique and could not be realized in the frame of our project.

4. Moreover, another verification of the theoretical nature in this time, was used and it concerns the assimilation of the cross-section of the microchannels $E$ to a circular cross-section and to calculate the equivalent hydraulic diameter. Using this technique of the hydraulic diameter calculation and the analytical expression of the mass flow rate for tubes, the velocity slip coefficient $\left(\sigma_{p}\right)$ and TMAC $(\alpha)$ were calculated and their new values seem to be more reasonable, i.e. in the interval determined by the Maxwell model. The resulting TMAC values are in the range $[0.9,1.0]$ for the microchannels $E 1$ and $E 2$ and are of the order of 0.7 and 0.6 for the microchannels $E 3$ and $E 4$, respectively. Low TMAC values obtained for the microchannels $E 3$ and $E 4$ are due to the large aspect ratio of these microchannels, hence, their assimilation to a circular section is not pertinent.

Finally, it was shown that the probable factor influencing the results of the microchannels $E$ is the gold layer takeoff inside the microchannels, which causes the change of their cross-section shape. The change of the microchannels crosssection induces that the theoretical model used here for rectangular channels is not adapted for the analysis. 


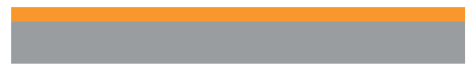

(a) Resin coating

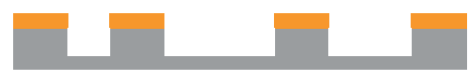

(c) DRIE etching

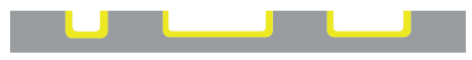

(e) Resin stripping and ion attack

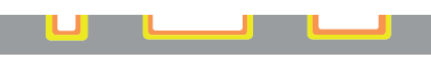

(g) Exposure and development

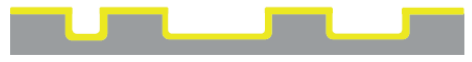

(i) Resin stripping

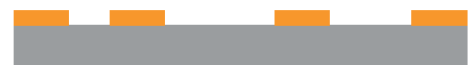

(b) Exposure and development

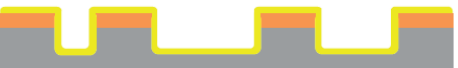

(d) Au or $\mathrm{SiO}_{2}$ deposit

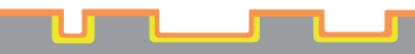

(f) Resin coating

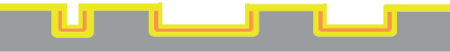

(h) Gold (Au) deposit (100 nm)

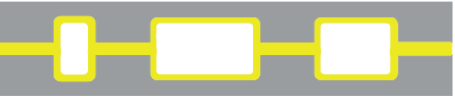

(j) Wafers bounding channel A \& S1

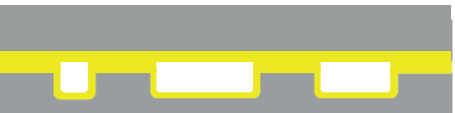

(j) Wafers bonding channels S \& E

Figure 1: Rectangular microchannels fabrication process. 


\begin{tabular}{|l|l|}
\hline \hline\|\| & Leveling: 2 zones \\
\hline \hline & \multicolumn{2}{|l|}{ Zoom: none } \\
\hline & \\
\hline & \\
\hline$R a$ & $0.001114 \mu \mathrm{m}$ \\
\hline $\mathrm{Rq}$ & $0.001443 \mu \mathrm{m}$ \\
\hline
\end{tabular}

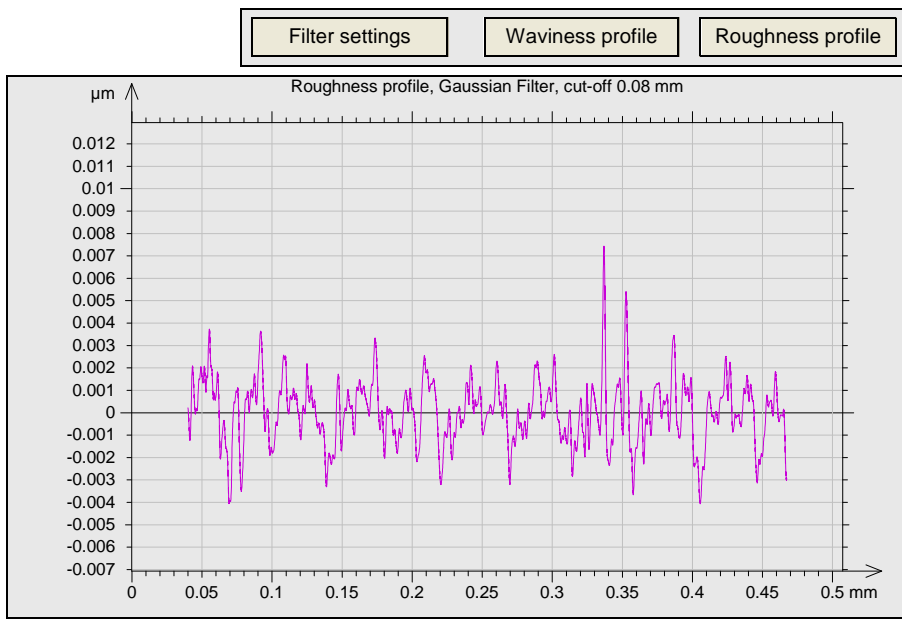

Figure 2: Graph of the roughness for the microchannel $S 3$.

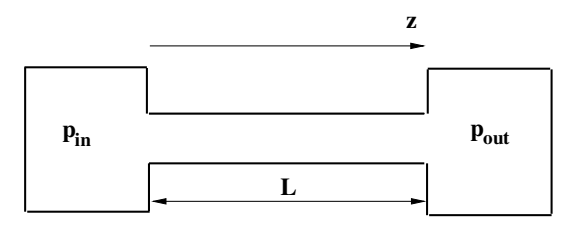

(a)

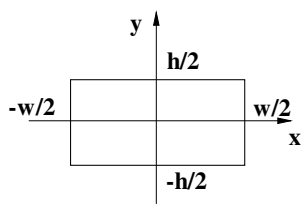

(b)

Figure 3: (a) Physical domain, (b) channel cross-section. 

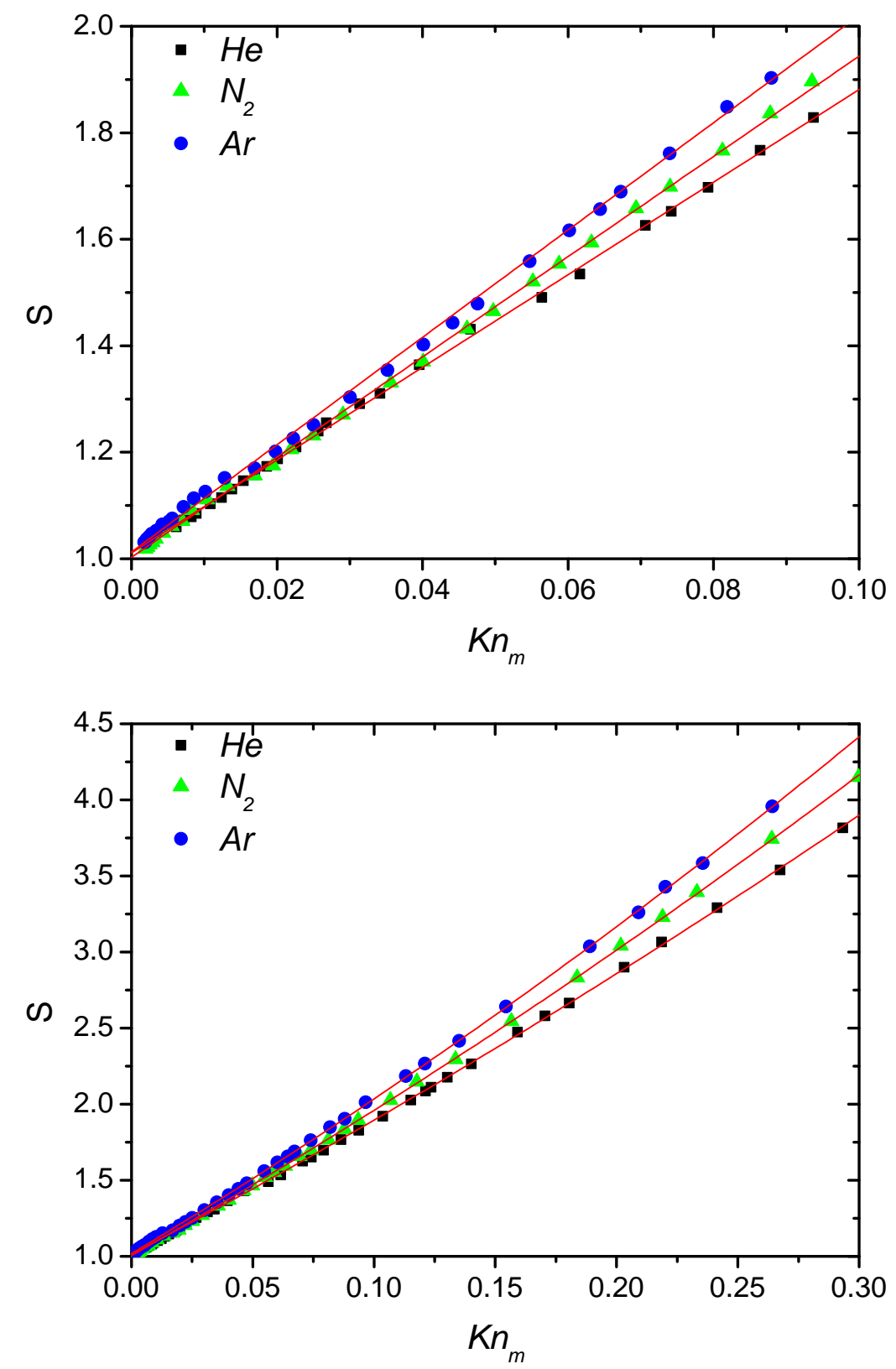

Figure 4: Dimensionless mass flow rate $S^{\exp }$ (44) plotted as function of the mean Knudsen number, fitted with first and second orders polynomial expressions for microchannel $A 3$, upper and down figure, respectively. 

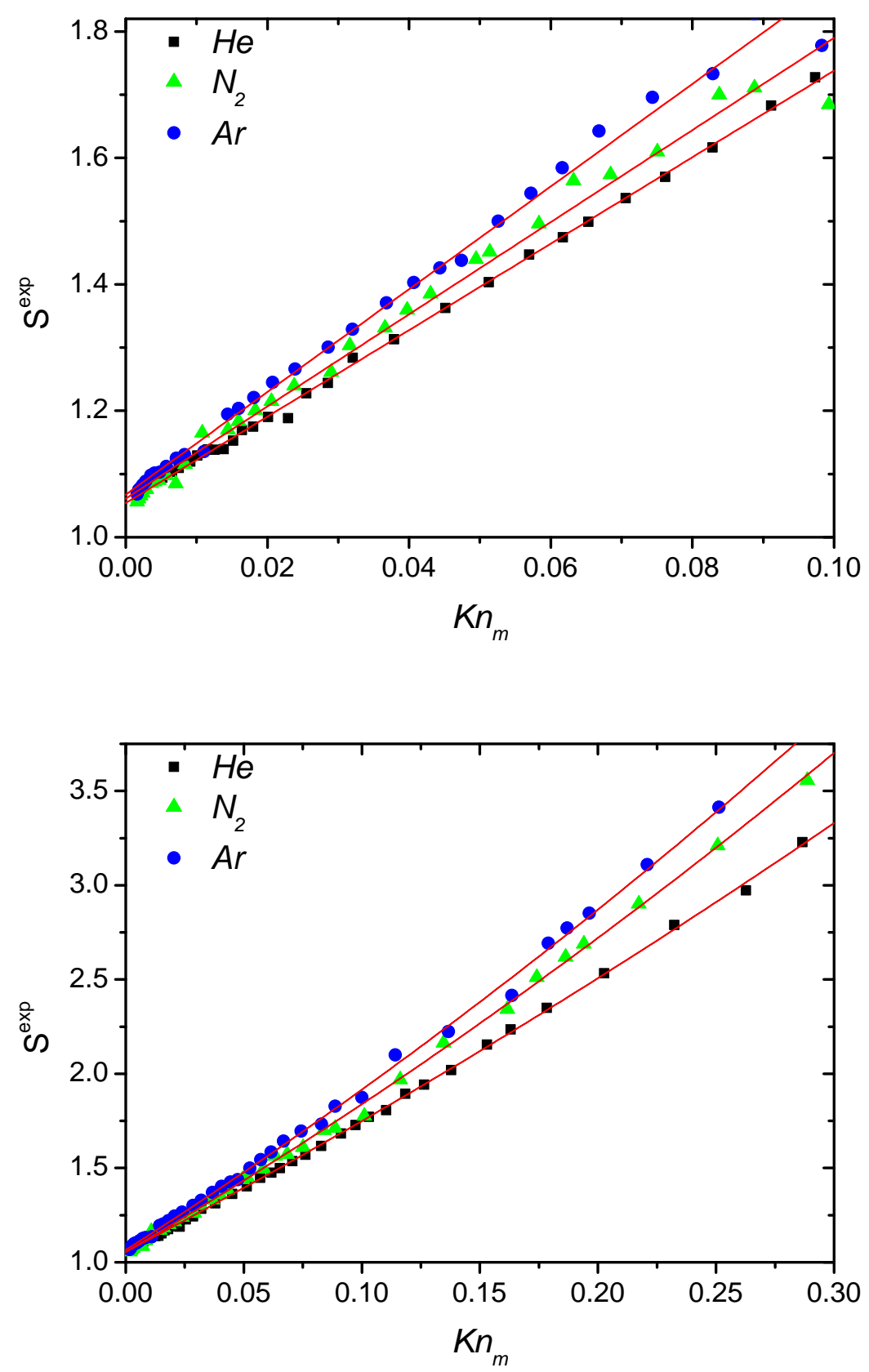

Figure 5: Dimensionless mass flow rate $S^{e x p}$ plotted as function of the mean Knudsen number, fitted with first and second order polynomial expressions on the upper and down plots, respectively, for the microchannels $E 4$. 

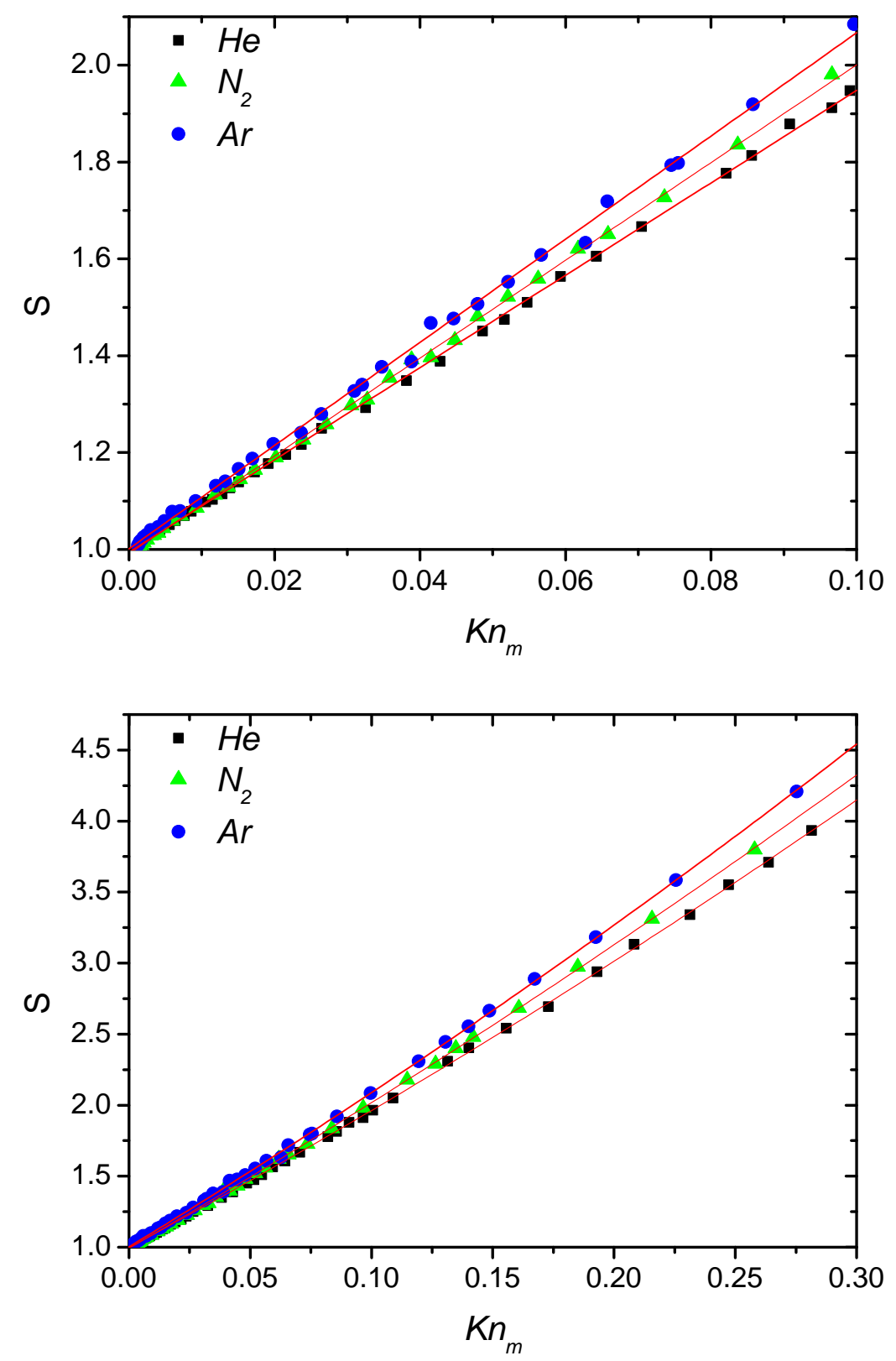

Figure 6: Dimensionless mass flow rate $S^{e x p}$ plotted as function of the mean Knudsen number, fitted with first and second order polynomial expressions, upper and down plots, respectively, for the microchannels $S 4$. 


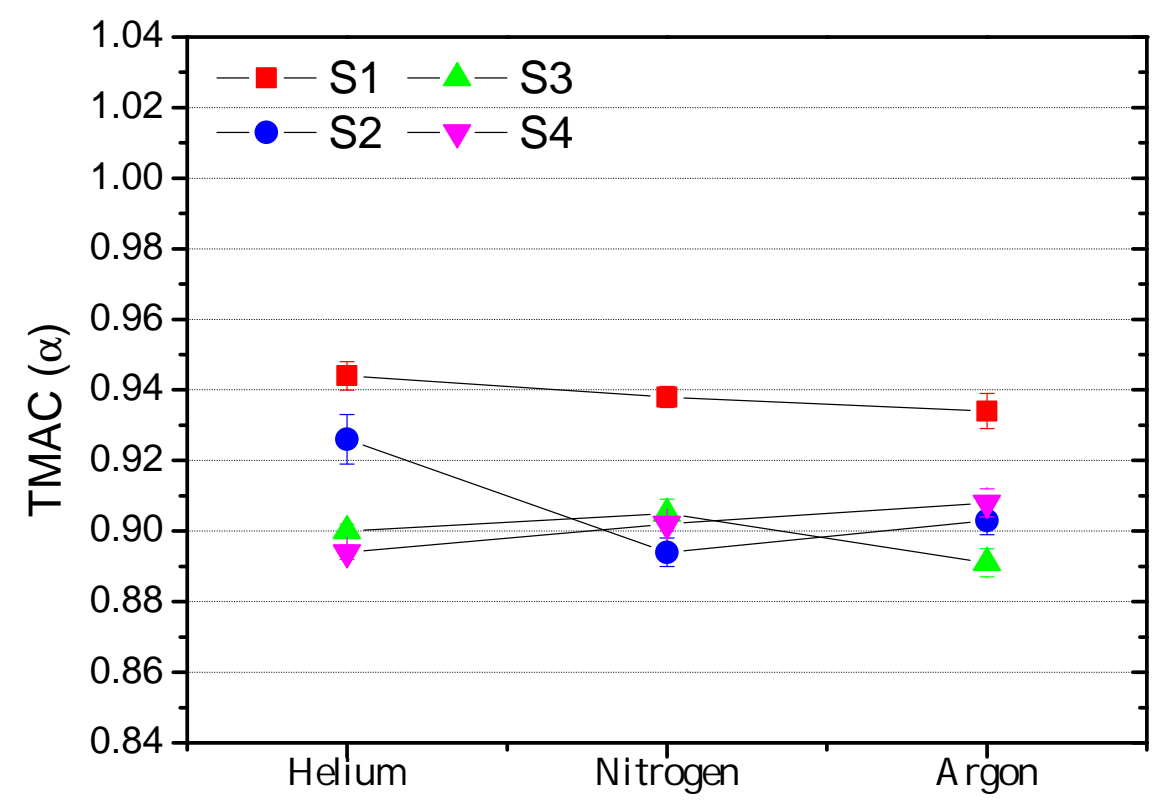

Figure 7: The TMAC for the microchannel of group $S$, obtained with the first order traitment. 